\title{
Forecast constraints on anisotropic stress in dark energy using gravitational waves
}

\author{
Weiqiang Yang ${ }^{\oplus},{ }^{1 \star}$ Supriya Pan, ${ }^{2 \star}$ David F. Mota ${ }^{3 \star}$ and Minghui Du ${ }^{4 \star}$ \\ ${ }^{1}$ Department of Physics, Liaoning Normal University, Dalian 116029, P. R. China \\ ${ }^{2}$ Department of Mathematics, Presidency University, 86/1 College Street, Kolkata 700073, India \\ ${ }^{3}$ Institute of Theoretical Astrophysics, University of Oslo, 0315 Oslo, Norway \\ ${ }^{4}$ Institute of Theoretical Physics, School of Physics, Dalian University of Technology, Dalian 116024, P. R. China
}

Accepted 2020 June 15. Received 2020 June 10; in original form 2020 March 29

\begin{abstract}
It is always interesting to investigate how well can a future experiment perform with respect to others (present or future ones). Cosmology is really an exciting field where a lot of puzzles are still unknown. In this paper, we consider a generalized dark energy (DE) scenario where anisotropic stress is present. We constrain this generalized cosmic scenario with an aim to investigate how gravitational waves standard sirens (GWSS) may constrain the anisotropic stress, which, according to the standard cosmological probes, remains unconstrained. In order to do this, we generate the luminosity distance measurements from $\mathcal{O}\left(10^{3}\right)$ mock GW events that match the expected sensitivity of the Einstein Telescope. Our analyses report that, first of all, GWSS can give better constraints on various cosmological parameters compared to the usual cosmological probes, but the viscous sound speed appearing due to the DE anisotropic stress is totally unconstrained even after the inclusion of GWSS.
\end{abstract}

Key words: cosmological parameters - dark energy - dark matter-large-scale structure of Universe.

\section{INTRODUCTION}

The thrilling chapter of modern cosmology begun with the late-time accelerating phase of our universe (Ade et al. 2016). This accelerated expansion is usually ascribed by the introduction of some exotic fluid with high negative pressure. This exotic fluid could be either some dark energy (DE) fluid (in the context of Einstein's general theory of gravity; Copeland, Sami \& Tsujikawa 2006) or some geometrical DE (coming from the modified gravity theories; Capozziello \& De Laurentis 2011; Nojiri \& Odintsov 2011; Nojiri, Odintsov \& Oikonomou 2017). In this paper, we confine ourselves into the first approach, which means the DE fluid. In other words, our discussions will be restricted to the Einstein's gravitational theory. Within the framework of general relativity, a cluster of DE models (see Copeland et al. 2006 for various DE models) have been introduced in the literature; however, most of them are basically the variations of either the cosmological constant or some scalar field theory (Wetterich 1988). These variations naturally include different kind of couplings between the matter components of the universe (Amendola 2000; Mota \& Shaw 2006, 2007), couplings to the gravitational sector (Koivisto \& Mota 2007a,b), or some non-canonical scalar field models, such as tachyons (Sen 2002a,b,c, 2003, 2005; Gibbons 2002), K-essence (Armendariz-Picon, Mukhanov \& Steinhardt 2001), etc. All such models are usually directed to explain the late accelerated expansion of the universe. Having a number of different DE models, it is quite natural to examine some important features of the DE models that might be able to discriminate between them, or, alternatively, such features might be able to provide some potential techniques that may act as a baseline to construct the DE models. Certainly, searching these features is indeed worth exploring based on the current cosmological research.

However, it has been already explored that in order to rule out some DE models or to discriminate between a number of existing DE models, the background evolution is not sufficient at all. The evolution of the models at the level of perturbations must be considered into the picture in order to have a wider understanding of the models. According to the theory of general relativity, any matter component with the equation of state $w \neq-1$, where $w=-1$ denotes the cosmological constant, must fluctuate. Thus, it is straightforward to realize that any DE model with $w \neq-1$ should have perturbations. However, it might happen that such perturbations could be small enough, depending on the nature of the equation of state $w$. For example, if the underlying DE fluid is smooth enough or if the Jeans length of this DE is large, its perturbations might be restricted to very large scales only. This kind of feature is exhibited in minimally coupled quintessence models because for such models, the sound speed of perturbations, $c_{\mathrm{s}}^{2}$, is equal to the speed of light, $c_{\mathrm{s}}^{2}=1$, which consequently sets a large Jeans length (Bean \& Dore 2004; Xia et al. 2008). While on the other hand, for some other DE candidates, this does not usually happen (Bagla, Jassal \& Padmanabhan 2003; Mota \& van de Bruck 2004), and, as a consequence, one could differentiate between the DE models. 
Moreover, aside from the equation of state $w$ and the sound speed of perturbations $c_{\mathrm{s}}^{2}$, one more important characteristic of a cosmic fluid is its anisotropic stress $\sigma$ (Hu 1998). Although for a class of cosmological models including minimally coupled scalar field and perfect fluids, the anisotropic stress vanishes, it is a generic property of realistic fluids with finite shear viscous coefficients (Schimd et al. 2007; Brevik et al. 2004). Let us note that while $w$ and $c_{\mathrm{s}}^{2}$, respectively, determine the background and perturbative evolutions of the underlying cosmic fluid that is rotationally invariant, the anisotropic stress $\sigma$ actually gives a quantification on how much the pressure of the cosmic fluid varies with the direction. In fact, the perturbation for anisotropic stress is very important for understanding the evolution of inhomogeneities in the early universe (Hu 1998; Koivisto \& Mota 2006; Mota et al. 2007). Thus, undoubtedly, it is a natural question to investigate whether the current observational data may indicate for a non-zero anisotropic stress perturbations in the DE-dominated late-accelerating universe.

The effects of anisotropic stress, which may appear due to possible viscosity of DE, have not been paid much attention in the literature. The reason for neglecting the anisotropic stress is that, for the conventional DE fluids, such as the cosmological constant or canonical scalar field models, $\sigma=0$. But that should not be a logical case since there is no such fundamental theory available yet, which could correctly describe the actual dynamics of DE; hence, the assumption of $\sigma=0$ for any DE model does not have any sense anymore. Therefore, from an unbiased scientific point of view, the presence of an anisotropic stress into the cosmic sector should be fairly considered and it is better to examine its non-null character, if any, with the help of recently available observational data. Some earlier analyses have shown that coupled scalar field models have a non-negligible anisotropic stress (Schimd et al. 2007). Additionally, DE vector field candidates (proposed in Armendariz-Picon 2004; Kiselev 2004) also allow non-zero anisotropic stress. Furthermore, some model-independent analyses (Pinho, Casas \& Amendola 2018; Arjona \& Nesseris 2020) have strongly argued that a non-null anisotropic stress in DE should be present according to the recent observational data. Therefore, it is fairly clear that the generalized DE models including the anisotropic stress should be investigated in detail and several investigators (see e.g. Saltas \& Kunz 2011; Sapone et al. 2013; Amendola et al. 2014; Cardona, Hollenstein \& Kunz 2014; Chang \& Xu 2014; Chang, Lu \& Xu 2014; Majerotto, Sapone \& Schäfer 2016) tried to model the anistropic stress in various ways as well as confronted it with the observational data. In particular, the role of an anisotropic stress was investigated in an interacting DE-DM scenario (Yang et al. 2019a).

In this paper, our approach is very appealing. We want to probe the anisotropic stress using the gravitational waves data, detected recently (Abbott et al. 2016a,b, 2017a,b,c). In particular, we shall use the simulated gravitational waves standard sirens (GWSS) data to constrain the anisotropic stress with an aim to what future cosmological probe can tell us. The simulated GWSS data have already proved its super constraining power applied recently to various cosmological models (see e.g. Di Valentino \& Melchiorri 2018; Di Valentino et al. 2018; Du et al. 2019; Yang et al. 2019b, 2020). In fact, gravitational waves data are believed to offer more information about the nature of dark matter, DE, and modified gravity theories (see e.g. Cai \& Zhang 2016; Maselli et al. 2016; Baker et al. 2017; Bettoni et al. 2017; Creminelli \& Vernizzi 2017; Ezquiaga \& Zumalacárregui 2017; Addazi, Cai \& Marciano 2018; Cai et al. 2018; Casalino et al. 2018; Chakraborty et al. 2018; Flauger \& Weinberg 2018; Liu et al. 2018; Oost, Mukohyama \& Wang 2018; Visinelli, Bolis \& Vagnozzi 2018; Zhao, Wright \& Li 2018). In the recent past, the effects of the GWSS data on various cosmological theories and related key parameters have been greatly studied and due to its growing interest in the cosmological community as reflected in a series of investigations performed by many investigators (Nunes, Pan \& Saridakis 2018; Shafieloo, Keeley \& Linder 2020; Wei 2018; Zhang et al. 2018; Casalino et al. 2019; Copeland et al. 2019; Bonilla et al. 2020; Belgacem et al. 2019; D’Agostino \& Nunes 2019; Kase \& Tsujikawa 2019; Lin et al. 2019; Nunes, Alves \& de Araujo 2019; Qiao et al. 2019; Zhao et al. 2019a,b; Zhao et al. 2020), it is clearly realized that GWSS are in the limelight of modern cosmology. Thus, the consideration of GWSS has significant effects on the dynamics of our universe. In this paper, we use the simulated GWSS data from the Einstein Telescope (Punturo et al. 2010) (see also a number of works focused on this specific telescope; Mishra et al. 2010; Sathyaprakash, Schutz \& Van Den Broeck 2010; Chassande-Mottin et al. 2011; Gair et al. 2011; Hannam \& Hawke 2011; Huerta \& Gair 2011a,b; Zhao et al. 2011; Regimbau et al. 2012; Sathyaprakash et al. 2012; Taylor \& Gair 2012; Cai \& Yang 2017; Zhang et al. 2017, 2019, 2020; Bachega et al. 2020); however, technically, one can equally consider other observatories like Laser Interferometer Space Antenna (LISA; Amaro-Seoane et al. 2017), Deci-hertz Interferometer Gravitational wave Observatory (DECIGO; Kawamura et al. 2011), and TianQin (Luo et al. 2016). In fact, it will be interesting to use different simulated GWSS data from all the above sources with an aim to a detailed investigations in this direction.

The work has been organized in the following manner. In Section 2, we review the parametrization of a generalized cosmic fluid and introduce its connection with anisotropic stress. In Section 3, we describe the observational data, namely both standard cosmological data and the simulated gravitational waves data. Then in Section 4, we describe the extracted results obtained after using varieties of observational (real and forecasted) data sets. Finally, in Section 5, we close this work with the main findings and comment on some future works that should be performed along these lines.

\section{PARAMETRIZING DE STRESS: FLRW BACKGROUND}

The energy momentum tensor of a general cosmic fluid is defined as (Koivisto \& Mota 2006; Mota et al. 2007)

$T_{\mu \nu}=\rho u_{\mu} u_{\nu}+p h_{\mu \nu}+\Sigma_{\mu \nu}$,

where $p$ and $\rho$ are, respectively, the pressure and energy density of the perfect fluid; $u_{\mu}$ is the four-velocity vector of this fluid, and $h_{\mu \nu}$, the projection tensor, is defined as $h_{\mu \nu} \equiv g_{\mu \nu}+u_{\mu} u_{\nu}$. The quantity $\Sigma_{\mu \nu}$ in (1) may include only spatial inhomogeneity that vanishes for a perfect fluid, which means $\Sigma_{\mu \nu} \equiv 0$. Additionally, for a homogeneous and isotropic universe, $\Sigma_{\mu \nu}$ is also zero at the level of background; in fact, in such a case, it denotes the anisotropic perturbation at the first order. Thus, at the background level, since $\Sigma_{\mu \nu}=0$, the evolution of the fluid 
(1) is determined by the continuity equation,

$\dot{\rho}+3 H(p+\rho)=0$,

where $H$ is the Hubble rate of the Friedmann-Lemaitre-Robertson-Walker (FLRW) universe. The condition for the adiabaticity of a fluid is $p=p(\rho)$, which tells us that the evolution of the sound speed is determined by the equation of state $w=p / \rho$, alone. However, being the most general, the sound speed is defined as the ratio of pressure to the density perturbations in the frame comoving with the DE fluid, defined as

$c_{\mathrm{s}, \mathrm{a}}^{2} \equiv \frac{\dot{p}}{\dot{\rho}}=w-\frac{\dot{w}}{3 \mathcal{H}(1+w)}$,

where an overhead dot represents the differentiation with respect to the conformal time $\tau$; $w$, being the equation of state, is defined as $w \equiv p / \rho$; and $\mathcal{H}=\dot{a} / a$ is the conformal Hubble parameter. We note that the relation between the perturbations of $\delta p$ and $\delta \rho$ is $\delta p=c_{\mathrm{s}, \mathrm{a}}^{2} \delta \rho$. However, for an entropic fluid, the pressure $p$ may not only depend on the energy density $\rho$. In fact, there might have another degree of freedom in order to describe the microphysical properties of the general cosmic fluid and such microphysical property is usually encoded in the effective speed of sound $c_{\mathrm{s}, \text { eff }}^{2}$, defined as

$\left.c_{\mathrm{s}, \mathrm{eff}}^{2} \equiv \frac{\delta p}{\delta \rho}\right|_{\mathrm{rf}}$,

in the rest frame ('rf') of the underlying cosmic fluid. In the absence of entropic perturbation, $c_{\mathrm{s}, \mathrm{eff}}^{2}=c_{\mathrm{s}, \mathrm{a}}^{2}$. Therefore, one may easily conclude that a perfect fluid is completely characterized by two quantities, one is its equation of state $w$ and the other is its effective speed of sound $c_{\mathrm{s}, \mathrm{eff}}^{2}$.

However, aside from the previous quantities, namely $w$ and $c_{\mathrm{s}, \text { eff }}^{2}$, associated with a cosmic fluid, one more important quantity is needed in order to understand the cosmic fluid at the level of background and perturbations. This quantity is the anisotropic stress $\sigma$, and it should be considered into the cosmological framework even in an isotropic and homogeneous FLRW universe, where the anisotropic stress $\sigma$ is, maybe, taken as the spatial perturbation. This anisotropic stress actually distinguishes between the Newtonian potential and curvature perturbation in the conformal Newtonian gauge.

So, now one can calculate the evolution equations at the level of perturbations for the above model considering any gauge. We choose the synchronous gauge in this paper and, using this gauge, the density perturbations and velocity perturbations can be written as (Ma \& Bertschinger 1995)

$\dot{\delta}=-(1+w)\left(\theta+\frac{\dot{h}}{2}\right)-3 \mathcal{H}\left(\frac{\delta p}{\delta \rho}-w\right) \delta$,

$\dot{\theta}=-\mathcal{H}\left(1-3 c_{\mathrm{s}, \mathrm{a}}^{2}\right)+\frac{\delta p / \delta \rho}{1+w} k^{2} \delta-k^{2} \sigma$,

where the anisotropic stress $\sigma$ is related to $\Sigma_{\mu \nu}$ (see equation 1) via $(\rho+p) \sigma \equiv-\left(\hat{k}_{i} \hat{k}_{j}-\delta_{i j} / 3\right) \Sigma^{i j}$. Now, using the effective speed of sound, one can recast the above equations as

$\dot{\delta}=-(1+w)\left(\theta+\frac{\dot{h}}{2}\right)+\frac{\dot{w}}{1+w} \delta-3 \mathcal{H}\left(c_{\mathrm{s}, \mathrm{eff}}^{2}-c_{\mathrm{s}, \mathrm{a}}^{2}\right)\left[\delta+3 \mathcal{H}(1+w) \frac{\theta}{k^{2}}\right]$,

$\dot{\theta}=-\mathcal{H}\left(1-3 c_{\mathrm{s}, \mathrm{eff}}^{2}\right) \theta+\frac{c_{\mathrm{s}, \mathrm{eff}}^{2}}{1+w} k^{2} \delta-k^{2} \sigma$,

where, following $\mathrm{Hu}$ (1998), we suppose that the anisotropic stress $\sigma$ satisfies the evolution equation

$\dot{\sigma}+3 \mathcal{H} \frac{c_{\mathrm{s}, \mathrm{a}}^{2}}{w} \sigma=\frac{8}{3} \frac{c_{\mathrm{vis}}^{2}}{1+w}\left(\theta+\frac{\dot{h}}{2}+3 \dot{\eta}\right)$,

where $c_{\mathrm{vis}}^{2}$ is the viscous speed of sound and it controls the relation between velocity/metric shear and the anisotropic stress. For a relativistic fluid, $c_{\mathrm{vis}}^{2}=1 / 3$. For any DE fluid, $c_{\mathrm{vis}}^{2}$ acts as a free model parameter to be determined by the observational data. In connection with that we recall a recent study (Arjona, Cardona \& Nesseris 2019), the authors argue that $c_{\mathrm{vis}}^{2}$ may have a dynamical nature, and this is an interesting issue that should be further investigated in light of the presently available potential cosmological probes. In the present, we shall, however, treat $c_{\text {vis }}^{2}$ to be a free parameter and following Huey (2004) and Koivisto \& Mota (2006), one notices that the value of $c_{\text {vis }}^{2} /(1+w)$ should be positive. This forces us to consider to different models, namely (i) the DE equation of state is in the quintessence regime, which means $-1<$ $w<0$ (Model I), and (ii) when the DE equation of state crosses the phantom divide line, which means, $w<-1$ (Model II). Let us note that for all the analyses that will be described in Section 4, we adopted the adiabatic initial conditions similar to Koivisto \& Mota (2006).

\section{STANDARD COSMOLOGICAL PROBES AND THE GWSS DATA}

We now proceed to extract the cosmological constraints using a set of usual DE probes and the simulated GWSS. In this section, we shall describe the standard cosmological data and then we will refer some works that describe the methodology to generate the mock GWSS. In what follows, we describe the standard cosmological probes first: 
Table 1. Flat priors set on various cosmological parameters for the statistical analysis.

\begin{tabular}{lcc}
\hline Parameter & Prior (Model I) & Prior (Model II) \\
\hline$\Omega_{\mathrm{b}} h^{2}$ & {$[0.005,0.1]$} & {$[0.005,0.1]$} \\
$\Omega_{\mathrm{c}} h^{2}$ & {$[0.01,0.99]$} & {$[0.01,0.99]$} \\
$\tau$ & {$[0.01,0.8]$} & {$[0.01,0.8]$} \\
$n_{\mathrm{S}}$ & {$[0.5,1.5]$} & {$[0.5,1.5]$} \\
$\log \left(10^{10} A_{\mathrm{s}}\right)$ & {$[2.4,4]$} & {$[2.4,4]$} \\
$100 \theta_{\mathrm{MC}}$ & {$[0.5,10]$} & {$[0.5,10]$} \\
$w$ & {$[-1,0]$} & {$[-3,-1]$} \\
$c_{\text {vis }}^{2}$ & {$[0,10]$} & {$[-10,0]$} \\
\hline
\end{tabular}

(i) CMB from Planck 2015: The cosmic microwave background (CMB) data are one of the potential data to probe the nature of DE. We use the Planck 2015 measurements (Adam et al. 2016; Planck Collaboration XI 2016) that include the high- and low- $\ell$ TT likelihoods (in the multipole range $2 \leq \ell \leq 2508$ ) plus the high- and low- $\ell$ polarization likelihoods. In this paper, we refer this data set as P15.

(ii) CMB from Planck 2018: We also consider the latest CMB data from Planck 2018 final release (Planck Collaboration VIII 2018; Planck Collaboration V 2019). In this paper, we refer this data set as P18.

(iii) $B A O$ : We include the baryon acoustic oscillations (BAO) distance measurements from different measurements (Beutler et al. 2011; Ross et al. 2015; Gil-Marín et al. 2016).

(iv) SN Ia: We use the latest compilation of Pantheon sample from Type Ia supernovae (SNe Ia; Scolnic et al. 2018). We note that SNe Ia data were the first astronomical data that signaled for the existence of some hypothetical DE fluid in the universe's sector. Thus, these particular data play a very important role in DE analysis.

The methodology to generate the GWSS is described by the present authors in Du et al. (2019) (also see Yang et al. 2020). This work deals with the same methodology as in Du et al. (2019) and Yang et al. (2020); thus, we avoid the repetition here and directly refer to Du et al. (2019) and Yang et al. (2020) for details. We would mark some important points here. Using the methodology here for this work, we generate 1000 simulated GW data for our purpose. The generation of $1000 \mathrm{GW}$ data is as follows. We first constrain the cosmological scenarios using the usual cosmological probes, such as CMB, BAO, and Pantheon. Then we use the best-fitting values of all the free and derived parameters obtained from the standard cosmological probes and assuming the present anisotropic DE models as the fiducial models, and following exactly the similar technique described in Du et al. (2019) and Yang et al. (2020), we generate the 1000 mock GW data. Next, we add these 1000 mock GW data to these standard cosmological probes and constrain the underlying cosmological scenarios. For the entire statistical analysis, we use the Markov chain Monte Carlo package cosmomC (Lewis \& Bridle 2002; Lewis 2013) equipped with a convergence diagnostic by Gelman-Rubin (Gelman \& Rubin 1992). The cosmomc code also supports the Planck 2015 Likelihood (Planck Collaboration XI 2016) and Planck 2018 likelihood (Planck Collaboration VIII 2018; Planck Collaboration V 2019). ${ }^{1}$ Finally, in Table 1, we enlist the flat priors on the cosmological parameters used during the time of statistical analysis.

\section{RESULTS}

In this section, we describe the main results on the cosmological parameters for the two variants of the model, namely Model I $(w>-1)$ and Model II $(w<-1)$, using various observational data sets. In particular, we focus on the effects of GW data on the cosmological parameters.

Before we present all the extracted cosmological constraints from the present cosmological scenarios, we wish to present the following. To generate GW catalogue, or mock GW data, it is essential to consider the fiducial model. Here, we consider Model I and Model II as the fiducial models. Now, assuming Model I as the fiducial model, we first constrain it using various observational data summarized in Table 2. Then considering the best-fitting values of all the free and derived parameters of this model (Model I), and following Du et al. (2019) and Yang et al. (2020), we generate the corresponding GW catalogue containing 1000 simulated GW events. In Fig. 1, we show $d_{L}(z)$ versus $z$ (with error bars on $d_{L}(z)$ for simulated $1000 \mathrm{GW}$ events. We use this catalogue as the forecasted data set and include them with the standard cosmological data sets, namely P15, BAO, and Pantheon, for the next step of the analysis. In a similar fashion, we generate simulated 1000 GW events for the second model in this work and Fig. 2 shows the corresponding $d_{L}(z)$ versus $z$ graphics. In what follows, we describe the observational constraints on each model considering the usual cosmological probes and the inclusion of the simulated GWSS.

4.1 Model I: $c_{\mathrm{vis}}^{2}>0, w>-1$

In Table 2, we show the constraints on the model parameters for the usual cosmological probes, and in Table 3, we display the constraints on the model parameters after the inclusion of the simulated GWs data with the usual cosmological probes. Thus, Tables 2 and 3 summarize the

\footnotetext{
${ }^{1}$ See http://cosmologist.info/cosmomc/ for a freely available code to extract the cosmological constraints.
} 
Table 2. The table displays the constraints on various free and derived cosmological parameters at 68 and 95 per cent CL for Model I using the usual cosmological probes, namely P15, BAO, and Pantheon.

\begin{tabular}{|c|c|c|c|c|}
\hline Parameters & $\mathrm{P} 15$ & $\mathrm{P} 15+\mathrm{BAO}$ & P15 + Pantheon & $\mathrm{P} 15+\mathrm{BAO}+$ Pantheon \\
\hline$\Omega_{\mathrm{c}} h^{2}$ & $0.1194_{-0.0015-0.0028}^{+0.0014+0.0029}$ & $0.1177_{-0.0011-0.0022}^{+0.0012+0.0021}$ & $0.1186_{-0.0012-0.0026}^{+0.0014+0.0025}$ & $0.1178_{-0.0010-0.0019}^{+0.0010+0.0019}$ \\
\hline$\Omega_{\mathrm{b}} h^{2}$ & $0.02223_{-0.00016-0.00030}^{+0.000}$ & $0.02236_{-0.00014-0.00028}^{+0.00014+0.00229}$ & $0.02230_{-0.00015-0.00029}^{+0.00015+0.00030}$ & $0.02236_{-0.00014-0.00028}^{+0.00014+0.00029}$ \\
\hline $100 \theta_{\mathrm{MC}}$ & $1.04073_{-0.00033-0.00066}^{+0.00033+0.00065}$ & $1.04097_{-0.00030-0.00059}^{+0.00030+0.00059}$ & $1.04083_{-0.00031-0.00061}^{+0.00031+0.00063}$ & $1.04096_{-0.00030-0.00060}^{+0.00030+0.00058}$ \\
\hline$\tau$ & $0.081_{-0.017-0.034}^{+0.017+0.033}$ & $0.089_{-0.017-0.032}^{+0.016+0.032}$ & $0.083_{-0.017-0.0033}^{+0.017+0.034}$ & $0.087_{-0.016-0.032}^{+0.016+0.033}$ \\
\hline$n_{\mathrm{S}}$ & $0.9654_{-0.0046-0.0090}^{+0.0045+0.0087}$ & $0.9702_{-0.0039-0.0075}^{+0.0040+0.0077}$ & $0.9677_{-0.0048-0.0088}^{+0.0042+0.0090}$ & $0.9700_{-0.0038-0.0076}^{+0.0037+0.0075}$ \\
\hline $\ln \left(10^{10} A_{\mathrm{s}}\right)$ & $3.095_{-0.033-0.065}^{+0.033+0.063}$ & $3.108_{-0.033-0.063}^{+0.032+0.063}$ & $3.098_{-0.033-0.066}^{+0.033+0.066}$ & $3.104_{-0.032-0.064}^{+0.032+0.064}$ \\
\hline$w$ & $<-0.854<-0.737$ & $<-0.953<-0.904$ & $<-0.973<-0.942$ & $<-0.974<-0.946$ \\
\hline$c_{v i s}^{2}$ & Unconstrained & Unconstrained & Unconstrained & Unconstrained \\
\hline$\Omega_{\mathrm{m} 0}$ & $0.348_{-0.037-0.048}^{+0.018+0.060}$ & $0.314_{-0.009-0.016}^{+0.007+0.017}$ & $0.315_{-0.009-0.017}^{+0.009+0.018}$ & $0.309_{-0.006-0.013}^{+0.006+0.013}$ \\
\hline$\sigma_{8}$ & $0.797_{-0.022-0.054}^{+0.032+0.048}$ & $0.820_{-0.0015-0.031}^{+0.017+0.010}$ & $0.823_{-0.0014-0.028}^{+0.014+0.017}$ & $0.824_{-0.014-0.028}^{+0.014+0.013}$ \\
\hline$H_{0}$ & $\begin{array}{c}64.11_{-1.70-4.93}^{+3.22+4.16} \\
\end{array}$ & $\begin{array}{l}66.98_{-0.66-1.72}^{+0.97+1.54} \\
\text { t. }\end{array}$ & $\begin{array}{c}67.09_{-0.67-1.55}^{+0.014-0.028} \\
\end{array}$ & $\begin{array}{l}-0.014-0.028 \\
67.51_{-0.52-1.16}^{+0.61+1.10}\end{array}$ \\
\hline
\end{tabular}

Note. For the DE equation of state, we present its upper limits at 68 and 95 per cent CL.
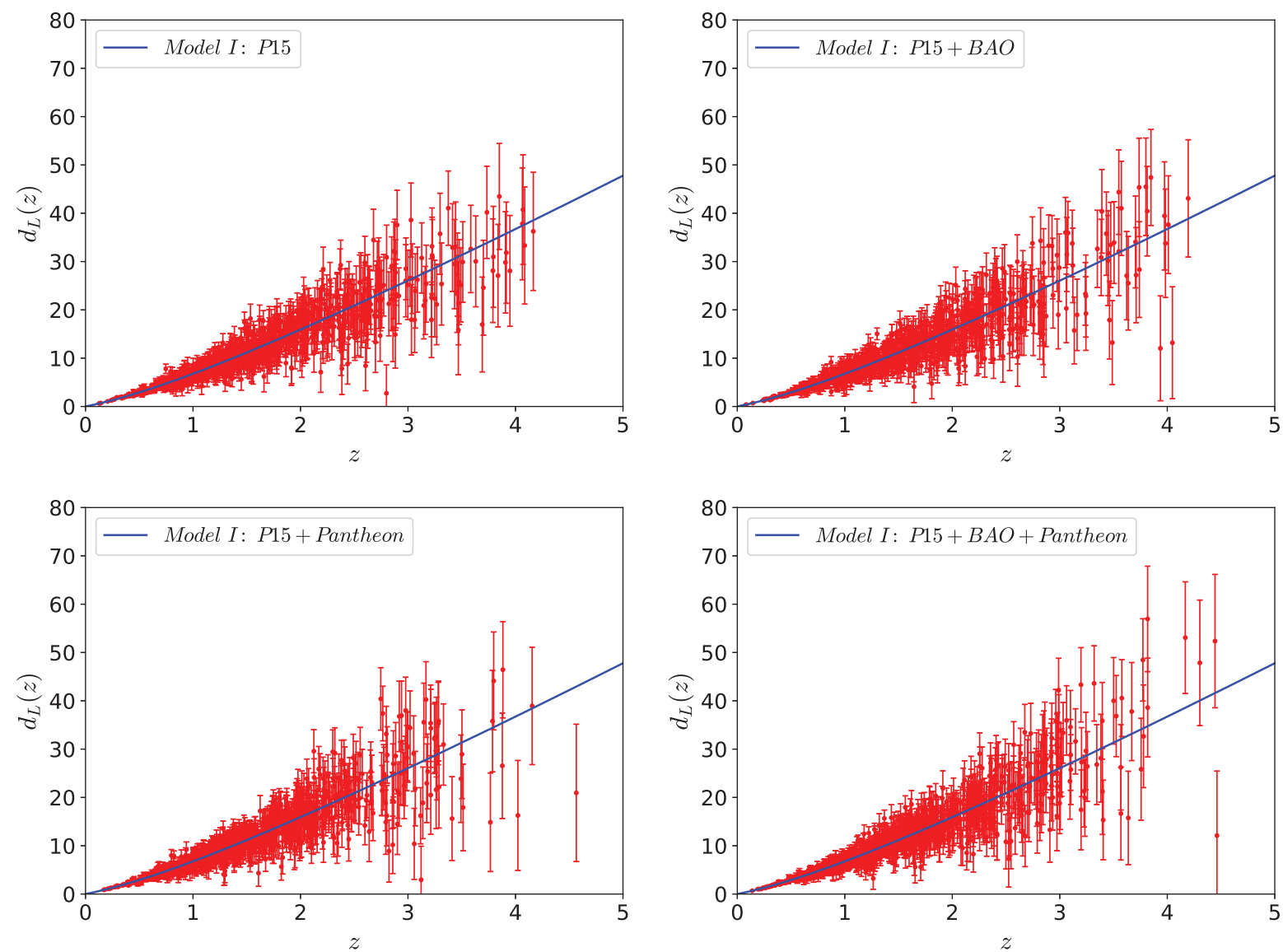

Figure 1. For the fiducial (Model I) model, we first constrain the cosmological parameters using the data sets P15, P15 + BAO, P15 + Pantheon, and P15 + $\mathrm{BAO}+$ Pantheon, and then we use the best fitting of the parameters for 'each data set' to generate the corresponding GW catalogue. Following this, in each panel, we show $d_{L}(z)$ versus $z$ catalogue with the corresponding error bars for 1000 simulated GW events. The upper left- and right-hand panels, respectively, present the catalogue $\left(z, d_{L}(z)\right)$ with the corresponding error bars for 1000 simulated events derived using the P15 alone and P15 + BAO data set. The lower left- and right-hand panels, respectively, present the catalogue $\left(z, d_{L}(z)\right)$ with the corresponding error bars for 1000 simulated events derived using the P15 + Pantheon and P15 + BAO + Pantheon data sets.

main results on this model. In the following, we become more explicit on the improvements of the constraints, if any, after the inclusion of GWs to the usual cosmological probes mentioned above.

Let us first focus on the constraints from P15 and P15 + GW. From Table 2, we notice that for P15 data alone, the Hubble constant at present, i.e. $H_{0}$, takes lower value compared to Lambda cold dark matter ( $\Lambda$ CDM)-based Planck but with high error bars compared to what we find in $\Lambda$ CDM-based Planck (Ade et al. 2016). In particular, one finds that for P15 alone, $H_{0}=64.11_{-1.70}^{+3.22}(68$ per cent CL). When the 
Table 3. In this table we show the constraints on various free and derived cosmological parameters of Model I at 68 and 95 per cent CL after the inclusion of GWSS data with the standard cosmological probes P15, BAO, and Pantheon.

\begin{tabular}{|c|c|c|c|c|}
\hline Parameters & $\mathrm{P} 15+\mathrm{GW}$ & $\mathrm{P} 15+\mathrm{BAO}+\mathrm{GW}$ & P15 + Pantheon + GW & $\begin{array}{c}\mathrm{P} 15+\mathrm{BAO}+ \\
\text { Pantheon }+\mathrm{GW}\end{array}$ \\
\hline$\overline{\Omega_{\mathrm{c}} h^{2}}$ & $0.1190_{-0.0010-0.0020}^{+0.0010+0.0018}$ & $0.1180_{-0.0011-0.0023}^{+0.0012+0.0021}$ & $0.1181_{-0.0009-0.0019}^{+0.0010+0.0017}$ & $0.1178_{-0.0009-0.0019}^{+0.0010+0.0018}$ \\
\hline$\Omega_{\mathrm{b}} h^{2}$ & $0.02226_{-0.00013-0.00024}^{+0.00012+0.00025}$ & $0.02233_{-0.00014-0.00027}^{+0.00014+0.00028}$ & $0.02234_{-0.00012-0.00024}^{+0.00012+0.00025}$ & $0.02235_{-0.00013-0.00025}^{+0.00012+0.00026}$ \\
\hline $100 \theta_{\mathrm{MC}}$ & $1.04078_{-0.00030-0.00058}^{+0.00029+0.0057}$ & $1.04092_{-0.00030-0.00059}^{+0.00031+0.00059}$ & $1.04092_{-0.000030-0.00057}^{+0.00030 .00058}$ & $1.04095_{-0.00029-0.00056}^{+0.00029+0.0056}$ \\
\hline$\tau$ & $0.082_{-0.016-0.031}^{+0.016+0.032}$ & $0.087_{-0.017-0.035}^{+0.017+0.032}$ & $0.086_{-0.016-0.032}^{+0.018+0.031}$ & $0.088_{-0.016-0.033}^{+0.018+0.031}$ \\
\hline$n_{\mathrm{s}}$ & $0.9667_{-0.0036-0.0069}^{+0.0037+0.0073}$ & $0.9692_{-0.0040-0.0078}^{+0.0040+0.0082}$ & $0.9691_{-0.0036-0.0068}^{+0.0036+0.0072}$ & $0.9700_{-0}^{+0.0036+0.0036-0.0070}$ \\
\hline $\ln \left(10^{10} A_{\mathrm{S}}\right)$ & $3.096_{-0.032-0.061}^{+0.032+0.063}$ & $3.104_{-0.033-0.067}^{+0.033+0.064}$ & $3.103_{-0.032-0.065}^{+0.033+0.062}$ & $3.105_{-0.031-0.067}^{+0.035+0.062}$ \\
\hline$w$ & $<-0.974<-0.948$ & $<-0.924<-0.901$ & $<-0.979<-0.957$ & $<-0.975<-0.950$ \\
\hline$c_{\mathrm{vis}}^{2}$ & Unconstrained & Unconstrained & Unconstrained & Unconstrained \\
\hline$\Omega_{\mathrm{m} 0}$ & $0.317_{-0.005-0.009}^{+0.005+0.009}$ & $0.319_{-0.006-0.010}^{+0.005+0.011}$ & $0.310_{-0.004-0.009}^{+0.004+0.009}$ & $0.309_{-0.004-0.008}^{+0.004+0.008}$ \\
\hline$\sigma_{8}$ & $0.824_{-0.014-0.027}^{+0.0014+0.027}$ & $0.815_{-0.015-0.030}^{+0.0015+0.030}$ & $0.825_{-0.014-0.027}^{+0.014+0.026}$ & $0.824_{-0.014-0.028}^{+0.014+0.027}$ \\
\hline$H_{0}$ & $66.94_{-0.38-0.80}^{+0.39+0.75}$ & $66.45_{-0.55-1.16}^{+0.63+1.13}$ & $67.48_{-0.35-0.73}^{+0.39+0.69}$ & $67.52_{-0.35-0.78}^{+0.42+0.70}$ \\
\hline
\end{tabular}

Note. For the DE equation of state, we present its lower limits at 68 and 95 per cent CL.
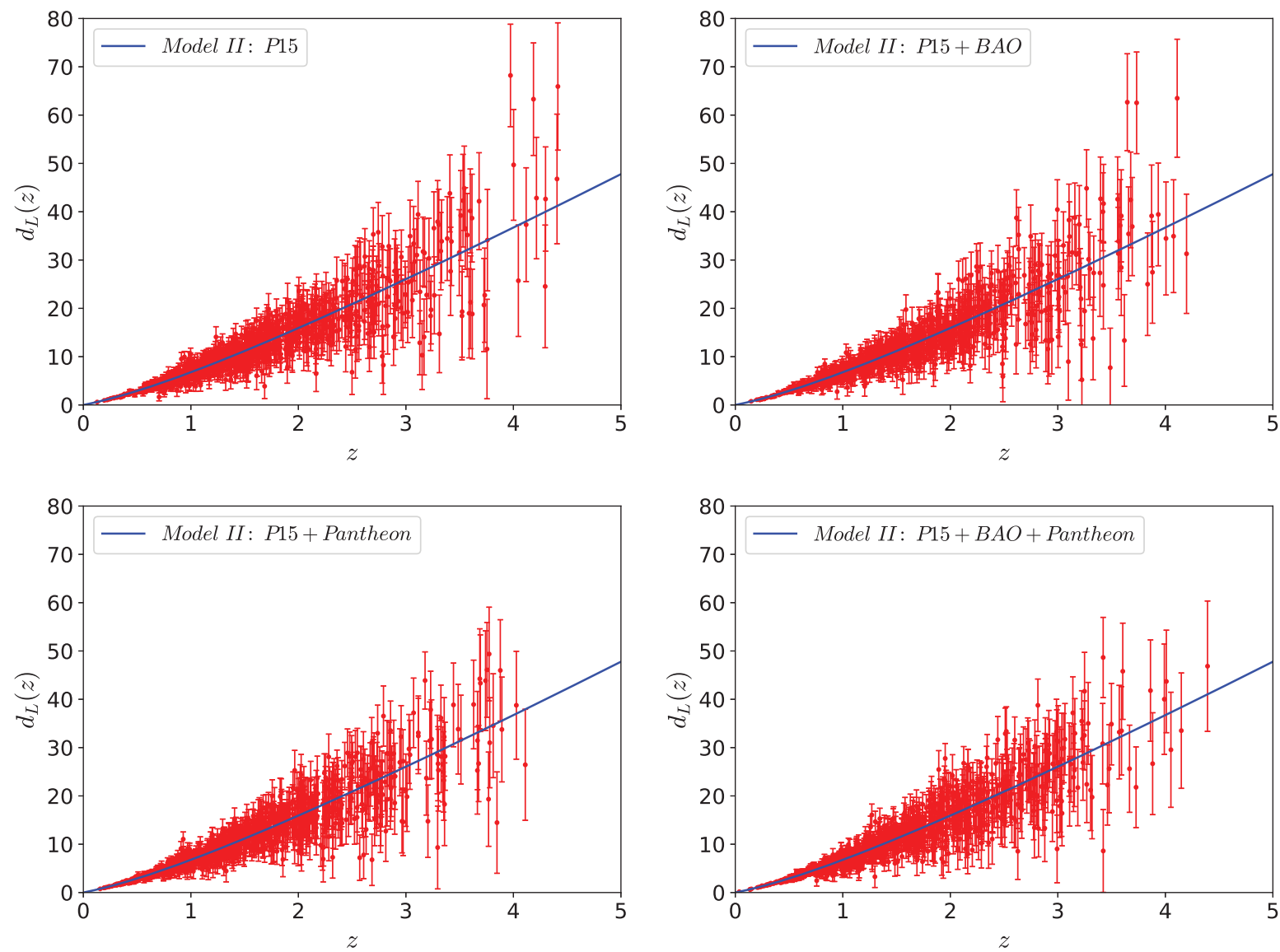

Figure 2. For the fiducial (Model II) model, we first constrain the cosmological parameters using the data sets P15, P15 + BAO, P15 + Pantheon, and P15 + $\mathrm{BAO}+$ Pantheon, and then we use the best fitting of the parameters for 'each data set' to generate the corresponding GW catalogue. Following this, in each panel, we show $d_{L}(z)$ versus $z$ catalogue with the corresponding error bars for 1000 simulated GW events. The upper left- and right-hand panels, respectively, present the catalogue $\left(z, d_{L}(z)\right)$ with the corresponding error bars for 1000 simulated events derived using the P15 alone and P15 + BAO data set. The lower left- and right-hand panels, respectively, present the catalogue $\left(z, d_{L}(z)\right)$ with the corresponding error bars for 1000 simulated events derived using the CMB + Pantheon and P15 + BAO + Pantheon data sets. 

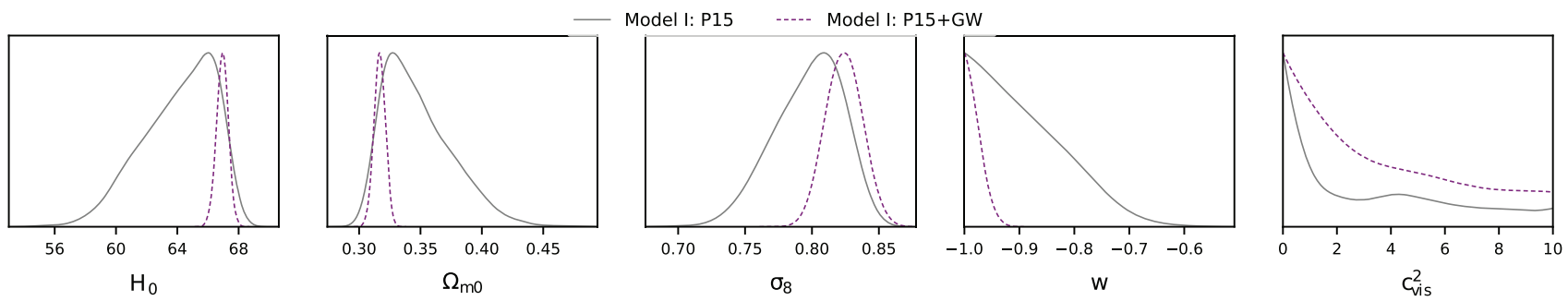

Figure 3. 1D marginalized posterior distributions of some key parameters of Model I for the data sets P15 and P15 + GW.
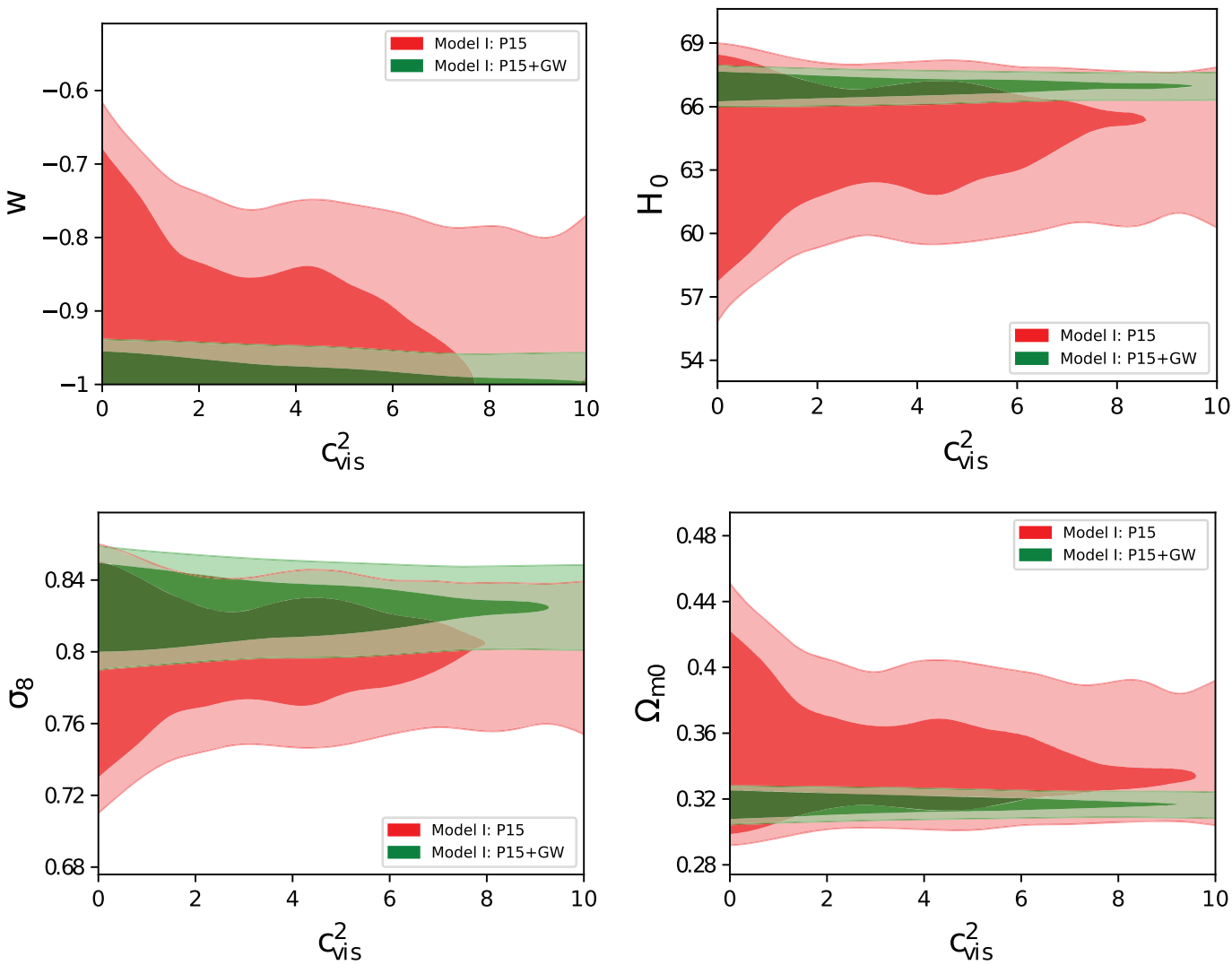

Figure 4. $2 \mathrm{D}$ contour plots showing the dependence of $c_{\mathrm{vis}}^{2}$ with other cosmological parameters for the P15 (red contours) and P15 $+\mathrm{GW}$ (green contours) data sets.

simulated GW data are added to P15, the Hubble constant rises up giving $H_{0}=66.94_{-0.38}^{+0.39}$ ( 68 per cent CL, P15 $+\mathrm{GW}$ ). One can clearly see that the inclusion of GW to P15 significantly reduces the error bars on $H_{0}$. In fact, the error bars on $H_{0}$ are reduced at least by a factor of 5 . This actually reflects the constraining power of GW. In a similar fashion, when we look at the other derived parameters of this model, namely $\Omega_{\mathrm{m} 0}, \sigma_{8}$, one can draw similar conclusion, which means the effects of GWs on the cosmological parameters is transparent. In fact, the free parameter, $w$, is also affected significantly after the addition of GW to P15. We see that the 68 per cent upper bound on $w$ for P15 alone is $w$ $<-0.854$, which is significantly changed to $w<-0.974$ after the addition of GW to P15. Now, concerning the viscous speed of sound, $c_{\mathrm{vis}}^{2}$, we find that this parameter is neither constrained by P15 alone nor the addition of GW to P15 helps to constrain it. We refer to Fig. 3 showing the 1D marginalized posterior distributions for some key parameters of this scenario for P15 and P15 + GW data sets. One may wonder that perhaps the increase of the prior on $c_{\mathrm{vis}}^{2}$ may help to constrain; however, this is not true in this case. We found that even if the prior varies in the interval $[0,100]$, this parameter remains unconstrained. We also show Fig. 4 displaying the dependence of $c_{\text {vis }}^{2}$ on other parameters for P15 and P15 + GW data sets. Thus, we find that even if we add mock GW data to CMB from P15, GW data do not add any extra constraining power to CMB from P15, which might constrain $c_{\text {vis }}^{2}$.

We now discuss the next two data sets, namely P15+BAO and its companion P15 + BAO + GW. For a quick view on the cosmological constraints, we refer to the third columns of both Tables 2 and 3. Additionally, for graphical views, we refer to the upper panel of Fig. 5, which shows the 1D marginalized posterior distributions for some selected parameters. We find that the addition of GW to P15 + BAO shifts the highest peak of $H_{0}$ towards higher values and shifts $\Omega_{\mathrm{m} 0}$ towards lower values. This is consistent since there is already a known correlation between $H_{0}$ and $\Omega_{\mathrm{m} 0}$. So, the addition of GW does not alter such correlation. A similar but very small shift of the $\sigma_{8}$ parameter is also observed. 


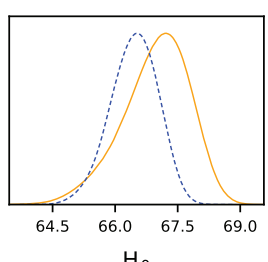

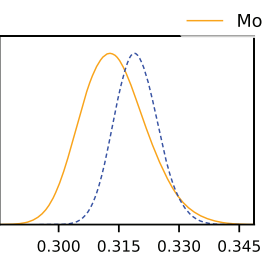

$\Omega_{\mathrm{mo}}$

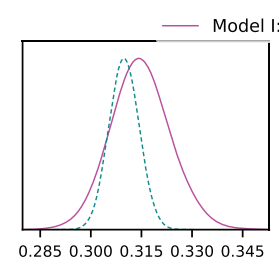

$\Omega_{\mathrm{mo}}$

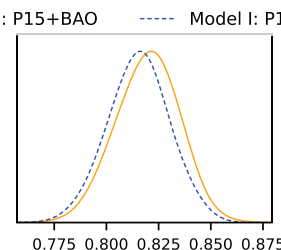

$\sigma_{8}$

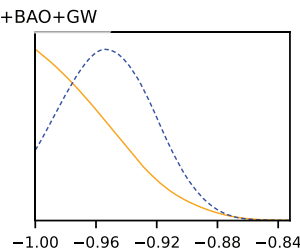

w

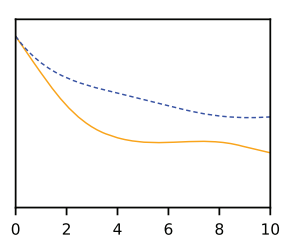

$c_{\text {vis }}^{2}$
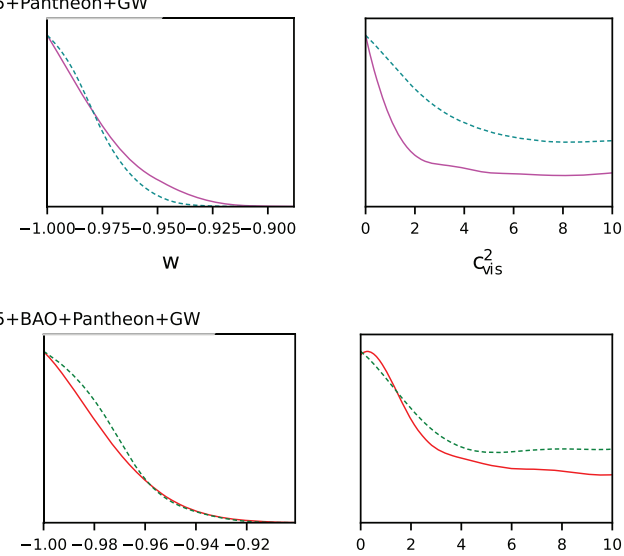

w

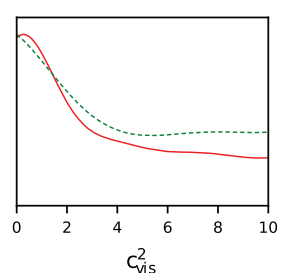

w

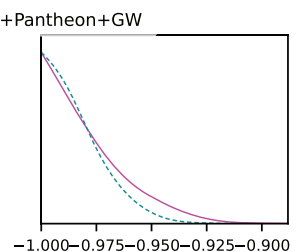

$\sigma_{8}$

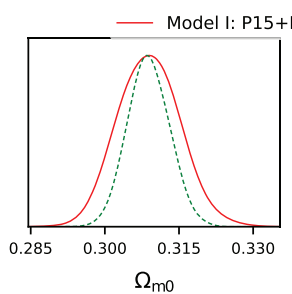

$\sigma_{8}$

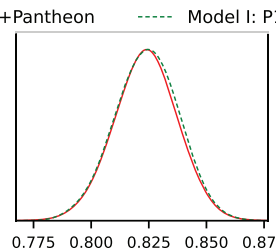

Figure 5. 1D marginalized posterior distributions of some key parameters of Model I for the data sets $\mathrm{P} 15+\mathrm{BAO}, \mathrm{P} 15+\mathrm{BAO}+\mathrm{GW}($ upper panel), $\mathrm{P} 15+$ Pantheon, P15 + Pantheon + GW (middle panel), and P15 + BAO+Pantheon, P15 + BAO + Pantheon + GW (lower panel).

Concerning the DE equation of state, $w$, we have an interesting observation. From the 1D posterior distribution of $w$ (upper panel of Fig. 5), we see that after the inclusion of GW to P15 + BAO, we find the highest peak of $w$, which was absent for the usual CMB + BAO analysis. Finally, we notice that the parameter $c_{\text {vis }}^{2}$ remains unconstrained for both the data sets, namely P15 $+\mathrm{BAO}$ and P15 $+\mathrm{BAO}+\mathrm{GW}$. So, we see that the addition of GW to $\mathrm{CMB}+\mathrm{BAO}$ does not alter the nature of this parameter.

We now consider the following two cases, namely CMB + Pantheon and $\mathrm{CMB}+$ Pantheon $+\mathrm{GW}$. The results are summarized in the fourth columns of both Tables 2 and 3. And we refer to the middle panel of Fig. 5 for a quick look on the 1D posterior distributions of some important parameter before and after the inclusion of GW to the corresponding data set (i.e. P15 + Patheon). Our results are very clear and straightforward. In a similar fashion to the previous two analyses (i.e. P15 + BAO and P15 + BAO + GW), here also we find that the addition of GW to P15 + Pantheon shifts the highest peak of the Hubble constant towards higher values having an additional shift of $\Omega_{\mathrm{m} 0}$ towards its lower values. However, the parameter space of both $H_{0}$ and $\Omega_{\mathrm{m} 0}$ is certainly improved due to GW. In addition, we do not find any changes to the parameter space of $\sigma_{8}$, which is clear if one looks at the 1D posterior distribution of this parameter. Moreover, we have a different result when one looks at the 1D posterior of $w$ for both CMB + Pantheon and P15 + Pantheon + GW. One could see that in contrast to the previous observation with P15 + BAO $+\mathrm{GW}$, the peak of $w$ disappears in this case. Finally, our conclusion regarding the viscous sound speed, $c_{\mathrm{vis}}^{2}$, remains the same, which means it is again unconstrained for both the data sets.

Lastly, we come to the last two data sets in this series, namely P15 + BAO + Pantheon and P15 + BAO + Pantheon + GW. The last columns of both Tables 2 and 3 summarize the constraints on the model parameters and the lower panel of Fig. 5 displays the 1D posterior distributions of some important parameters of this model. Looking at the lower panel of Fig. 5, specially for $H_{0}$ and $\Omega_{\mathrm{m} 0}$, we find their improvements after the inclusion of GW; however, we do not find any shifts of the highest peaks of $H_{0}$ and $\Omega_{\mathrm{m} 0}$ in their posterior distributions in contrary to the earlier cases, such as P15 + BAO + GW and P15 + Pantheon + GW. Here we again see that the parameter $c_{\mathrm{vis}}^{2}$ is still unconstrained for both the data sets. So GW data seem to be unable to constrain this particular parameter. One can try to understand this unconstrained nature of the viscous sound speed through Fig. 6 where we have displayed how various values of $c_{\text {vis }}^{2}$ can affect the temperature anisotropy in the CMB spectra. The left-hand panel of Fig. 6 shows the CMB TT spectra, while in the right-hand panel of Fig. 6, we have shown the corresponding residual plot. As one can see, in the lower multipoles region $(l<10)$, a very mild deviation in the CMB TT spectra from $c_{\mathrm{vis}}^{2}=0$ appears (see the right-hand panel of Fig. 6) due to the Integrated Sachs-Wolfe (ISW) effects coming from non-zero anisotropic stress in DE; see also (Chang \& Xu 2014).

When we finished all the analyses of this paper, Planck released its final CMB data (Planck Collaboration VIII 2018; Planck Collaboration V 2019). We then wanted to check whether the new CMB data from Planck 2018 final release (P18 as referred in the text) could constrain the parameter, $c_{\mathrm{vis}}^{2}$, of this scenario. We found that P18 data are also unable to constrain $c_{\mathrm{vis}}^{2}$, which is also unconstrained by the earlier P15 data. To illustrate this nature, in Fig. 7, we have shown the 1D posterior distributions of some key parameters of this model showing the constraining power of P18 data alone and also the effects of GW on $c_{\text {vis }}^{2}$ after its inclusion to P18. From the present analyses, we have clearly visualized that the unconstrained nature of $c_{\text {vis }}^{2}$ is not controlled by any of the employed external data sets, such as BAO, Pantheon, etc. And we have seen 

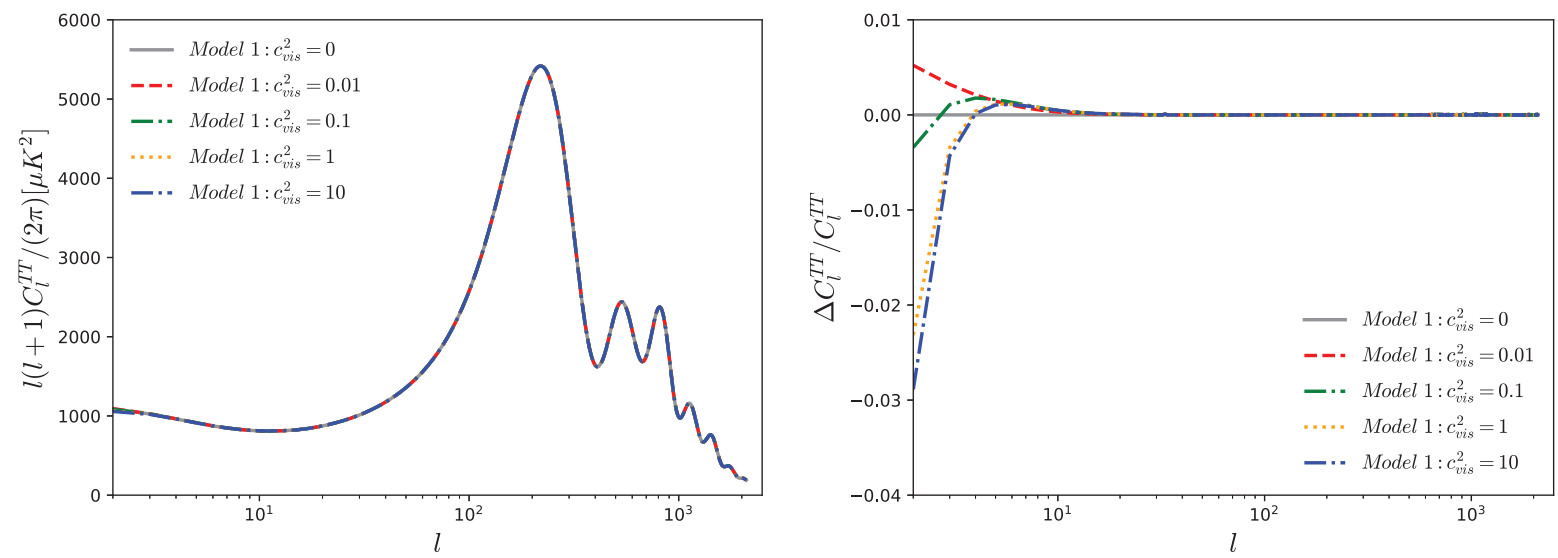

Figure 6. The plots reflect the effects on the CMB TT spectra for Model I taking various values of the viscous sound speed, $c_{\mathrm{vis}}^{2}$. As Model I restricts the $\mathrm{DE}$ equation of state, $w$ in the quintessence region, so we fix $w=-0.95$ and the remaining parameters have been taken from the combined analysis P15 + $\mathrm{BAO}+$ Pantheon (see the last column of Table 2). Note that the qualitative behaviour of the plots do not change if one varies the DE equation of state. From the right-hand panel, one can see that as long as $c_{\mathrm{vis}}^{2}$ increases, a very mild deviation of the curves with $c_{\mathrm{vis}}^{2} \neq 0$ from the curve representing $c_{\mathrm{vis}}^{2}=0$ (no anisotropic stress) appears.
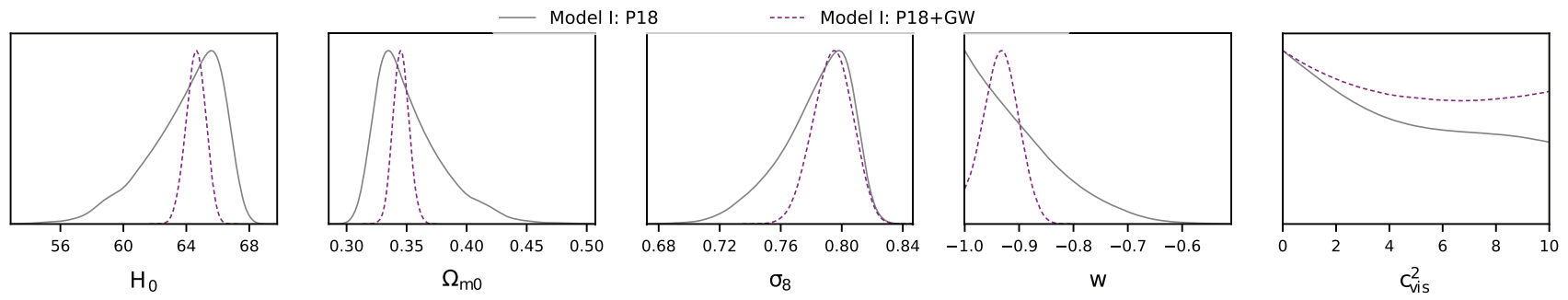

Figure 7. 1D marginalized posterior distributions of some key parameters of Model I for the data sets P18 and P18 + GW.

Table 4. The table presents the constraints on various free and derived cosmological parameters at 68 and 95 per cent CL for Model II using the usual cosmological probes, namely P15, BAO, and Pantheon.

\begin{tabular}{|c|c|c|c|c|}
\hline Parameters & P15 & $\mathrm{P} 15+\mathrm{BAO}$ & P15 + Pantheon & $\mathrm{P} 15+\mathrm{BAO}+$ Pantheon \\
\hline$\Omega_{\mathrm{c}} h^{2}$ & $0.1191_{-0.0014-0.0027}^{+0.0014+0.0027}$ & $0.1191_{-0.0011-0.0021}^{+0.0011+0.0022}$ & $0.1195_{-0.0013-0.0026}^{+0.0014+0.0026}$ & $0.1189_{-0.0011-0.0022}^{+0.0010+0.0022}$ \\
\hline$\Omega_{\mathrm{b}} h^{2}$ & $0.02228_{-0.00016-0.00030}^{+0.00016+0.00031}$ & $0.02226_{-0.00015-0.00028}^{+0.00014+0.00029}$ & $0.02224_{-0.00014-0.00029}^{+0.00014+0.00029}$ & $0.02228_{-0.00015-0.00027}^{+0.00014+0.00029}$ \\
\hline $100 \theta_{\mathrm{MC}}$ & $1.04080_{-0.00033-0.00063}^{+0.00033+0.00062}$ & $1.04080_{-0.00031-0.00059}^{+0.00030+0.00062}$ & $1.04073_{-0.00032-0.00062}^{+0.00031+0.00064}$ & $1.04083_{-0.00031-0.00060}^{+0.00031+0.00062}$ \\
\hline$\tau$ & $0.076_{-0.017-0.034}^{+0.018+0.034}$ & $0.080_{-0.017-0.032}^{+0.017+0.033}$ & $0.078_{-0.017-0.032}^{+0.017+0.033}$ & $0.082_{-0.017-0.032}^{+0.017+0.033}$ \\
\hline$n_{\mathrm{s}}$ & $0.9665_{-0.0046-0.0089}^{+0.0046+0.0093}$ & $0.9665_{-0.0040-0.0079}^{+0.0040+0.0077}$ & $0.9655_{-0.0044-0.0086}^{+0.0044+0.0087}$ & $0.9671_{-0.0039-0.0078}^{+0.0042+0.0077}$ \\
\hline $\ln \left(10^{10} A_{\mathrm{s}}\right)$ & $3.085_{-0.034-0.066}^{+0.034+0.066}$ & $3.093_{-0.033-0.062}^{+0.033+0.064}$ & $3.091_{-0.034-0.064}^{+0.033+0.065}$ & $3.095_{-0.032-0.065}^{+0.033+0.063}$ \\
\hline$w$ & $>-1.917>-1.973$ & $>-1.071>-1.133$ & $>-1.052>-1.088$ & $>-1.041>-1.078$ \\
\hline$c_{\mathrm{vis}}^{2}$ & Unconstrained & Unconstrained & Unconstrained & Unconstrained \\
\hline$\Omega_{\mathrm{m} 0}$ & $0.195_{-0.055-0.063}^{+0.020+0.088}$ & $0.296_{-0.008-0.020}^{+0.011+0.018}$ & $0.303_{-0.009-0.018}^{+0.009+0.018}$ & $0.301_{-0.007-0.014}^{+0.007+0.013}$ \\
\hline$\sigma_{8}$ & $0.989_{-0.052-0.139}^{+0.094+0.116}$ & $0.845_{-0.019-0.034}^{+0.016+0.036}$ & $0.841_{-0.015-0.029}^{+0.015+0.030}$ & $0.839_{-0.015-0.029}^{+0.015+0.029}$ \\
\hline$H_{0}$ & $86.69_{-6.03-16.53}^{+12.21+14.14}$ & $69.25_{-1.38-2.01}^{+0.80+2.33}$ & $68.57_{-0.97-1.70}^{+0.80+1.73}$ & $68.64_{-0.76-1.32}^{+0.60+1.40}$ \\
\hline
\end{tabular}

Note. For the DE equation of state, we present its lower limits at 68 and 95 per cent CL.

that when P15 data are unable to constrain $c_{\text {vis }}^{2}$; this parameter remains unconstrained by the external data sets. Thus, since P18 data remain unable to constrain $c_{\text {vis }}^{2}$, there is no reason to consider other external data sets with P18 in order to check whether the viscous sound speed will be constrained or not. Hence, we do not consider these combinations for this work. The nature of $c_{\text {vis }}^{2}$ will actually be the same in this case.

\subsection{Model II: $c_{\mathrm{vis}}^{2}<0, w<-1$}

In Table 4, we show the constraints on the model parameters using the usual cosmological probes, and in Table 5, we show the constraints on the model parameters after the inclusion of the simulated GWs data to the usual cosmological probes. Thus, Tables 4 and 5 summarize the main results on this model. 
Table 5. In this table, we show the constraints on various free and derived cosmological parameters of Model II at 68 and 95 per cent CL after the inclusion of GWSS data with the standard cosmological probes P15, BAO, and Pantheon.

\begin{tabular}{|c|c|c|c|c|}
\hline Parameters & $\mathrm{P} 15+\mathrm{GW}$ & $\mathrm{P} 15+\mathrm{BAO}+\mathrm{GW}$ & $\mathrm{P} 15$ + Pantheon + GW & $\begin{array}{c}\mathrm{P} 15+\mathrm{BAO}+ \\
\text { Pantheon }+\mathrm{GW}\end{array}$ \\
\hline$\Omega_{\mathrm{c}} h^{2}$ & $0.1184_{-0.0012-0.0023}^{+0.0012+0.0024}$ & $0.1194_{-0.0011-0.0019}^{+0.0010+0.0020}$ & $0.1196_{-0.0012-0.0021}^{+0.0011+0.0023}$ & $0.1184_{-0.0010-0.0020}^{+0.0009+0.0020}$ \\
\hline$\Omega_{\mathrm{b}} h^{2}$ & $0.02235_{-0.00014-0.00027}^{+0.00014+0.00027}$ & $0.02220_{-0.00013-0.00026}^{+0.00013+0.00026}$ & $0.02222_{-0.00013-0.00025}^{+0.00013+0.00025}$ & $0.02233_{-0.00013-0.00026}^{+0.00013+0.00025}$ \\
\hline $100 \theta_{\mathrm{MC}}$ & $1.04091_{-0.00030-0.00061}^{+0.00030+0.0059}$ & $1.04069_{-0.00031-0.00061}^{+0.00030+0.00059}$ & $1.04074_{-0.00030-0.00059}^{+0.00030+0.00057}$ & $1.04089_{-0.00029-0.000057}^{+0.00029+0.0058}$ \\
\hline$\tau$ & $0.085_{-0.017-0.033}^{+0.017+0.033}$ & $0.078_{-0.016-0.033}^{+0.016+0.031}$ & $0.078_{-0.016-0.033}^{+0.016+0.032}$ & $0.083_{-0.016-0.033}^{+0.016+0.031}$ \\
\hline$n_{\mathrm{s}}$ & $0.9683_{-0.0042-0.0085}^{+0.0043+0.0080}$ & $0.9657_{-0.0037-0.0073}^{+0.0037+0.0073}$ & $0.9652_{-0.0039-0.0078}^{+0.0042+0.0073}$ & $0.9683_{-0.0038-0.0079}^{+0.0038+0.0073}$ \\
\hline $\ln \left(10^{10} A_{\mathrm{s}}\right)$ & $3.101_{-0.033-0.065}^{+0.033+0.064}$ & $3.089_{-0.031-0.064}^{+0.031+0.061}$ & $3.090_{-0.033-0.064}^{+0.032+0.062}$ & $3.098_{-0.032-0.066}^{+0.035+0.066}$ \\
\hline$w$ & $>-1.084>-1.114$ & $>-1.055>-1.093$ & $>-1.06>-1.091$ & $>-1.046>-1.075$ \\
\hline$c_{v i s}^{2}$ & Unconstrained & Unconstrained & Unconstrained & Unconstrained \\
\hline$\Omega_{\mathrm{m} 0}$ & $0.292_{-0.004-0.008}^{+0.004+0.008}$ & $0.303_{-0.005-0.010}^{+0.006+0.010}$ & $0.304_{-0.005-0.010}^{+0.005+0.010}$ & $0.299_{-0.004-0.008}^{+0.004+0.008}$ \\
\hline$\sigma_{8}$ & $0.847_{-0.016-0.030}^{+0.015+0.031}$ & $0.840_{-0.015-0.029}^{+0.015+0.029}$ & $0.841_{-0.014-0.028}^{+0.014+0.028}$ & $0.838_{-0.015-0.030}^{+0.015+0.029}$ \\
\hline$H_{0}$ & $69.63_{-0.57-0.94}^{+0.48+1.05}$ & $68.52_{-0.67-1.08}^{+0.48+1.18}$ & $68.52_{-0.59-1.04}^{+0.49+1.09}$ & $68.83_{-0.50-0.82}^{+0.41+0.93}$ \\
\hline
\end{tabular}

Note. For the DE equation of state, we present its lower limits at 68 and 95 per cent CL.
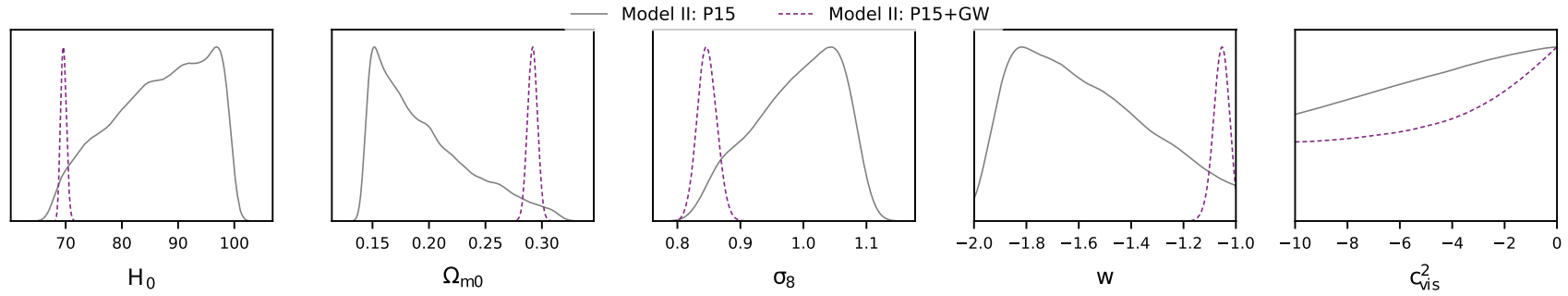

Figure 8. 1D marginalized posterior distributions of some key parameters of Model II for the data sets P15 and P15 + GW.

Now, following the similar pattern as with Model I, we analyse this model as well. Thus, we first focus on the data sets, namely P15 and P15 + GW, and discuss how the GWs data could improve the constraints on various free and derived parameters of this model. Looking at the constraint on $H_{0}$, one can quickly realize the effects of GWs on to it. From P15 alone, $H_{0}=86.69_{-6.03}^{+12.21}$ (68 per cent CL), while when GWs are added to P15, then $H_{0}$ is reduced both in its mean values as well as in its error bars, $H_{0}=69.63_{-0.57}^{+0.48}$ ( 68 per cent CL, P15 $+\mathrm{GW}$ ). In fact, the error bars on $H_{0}$ are reduced by a factor of more than 10. Thus, a real effect on the $H_{0}$ parameter for the introduction of GWs data is clearly visible. Similar effects on other cosmological parameters are equally evident. As one can see, the equation of state for DE is significantly improved after the addition of GWs to P15. More explicitly, the lower limit (at 68 per cent CL) of the DE equation of state changes from $w$ $>-1.917$ (P15) to $w>-1.084$ (P15 $+\mathrm{GW}$ ). Thus, looking at the constraints on $w$, one can clearly conclude that the inclusion of GWs to P15 not only decreases the mean values of $w$ taken for P15 data alone, but also this reduces the error bars of $w$ that arise from P15 data only. Finally, we come to the most important part of this work, namely the behaviour of $c_{\mathrm{vis}}^{2}$. We found that neither CMB nor P15 $+\mathrm{GW}$ could constrain this parameter. An exactly similar conclusion has been found for Model I. So, irrespective of the DE equation of state, this parameter remains constrained. In Fig. 8, we show the 1D marginalized posterior distributions of some important parameters discussed just above for the P15 and P15 + GW data sets. Moreover, in order to explicitly present the behaviour of $c_{\text {vis }}^{2}$ with other parameters, we present the 2D joint contours in Fig. 9.

For rest of the analyses with other cosmological data sets, we refer to Fig. 10 showing the 1D marginalized posterior distributions of some selected parameters. In particular, the upper panel of Fig. 10 compares the constraints on the model parameters for P15 + BAO and its companion P15 + BAO + GW. The lower panel of Fig. 10 similarly compares the constraints from P15 + Pantheon and P15 + Pantheon + GW and, finally, the last panel of Fig. 10 compares the constraints of some selective model parameters for P15 + BAO + Pantheon and P15 + BAO + Pantheon + GW. From Fig. 10, it is clear that due to the inclusion of GW to the standard cosmological data sets, some of the model parameters are affected; for instance, the effects on $H_{0}$ and $\Omega_{\mathrm{m} 0}$ are pretty clear while the effects on $w$ are not so pronounced much. However, the parameter on which we concentrate our focus in this work, namely $c_{\mathrm{vis}}^{2}$, is still unconstrained irrespective of either the usual cosmological probes or the inclusion of GW to them. So, effectively, even if we include the GW data into the standard cosmological probes, this specific parameter remains unconstrained, which means GW fails to constrain it. Similar to Model I, in Fig. 11, we show the temperature anisotropy in the CMB spectra for various values of $c_{\text {vis }}^{2}$. The left-hand panel of Fig. 11 shows the CMB TT spectra whilst the right-hand panel of Fig. 11 shows the corresponding residual plot. Similarly, we notice a very mild deviation in the CMB TT spectra in the lower multipoles region $(l<$ 10) from $c_{\text {vis }}^{2}=0$ (see the right-hand panel of Fig. 6) due to the ISW effects coming from non-zero anisotropic stress in DE; also see Chang \& $\mathrm{Xu}(2014)$. 

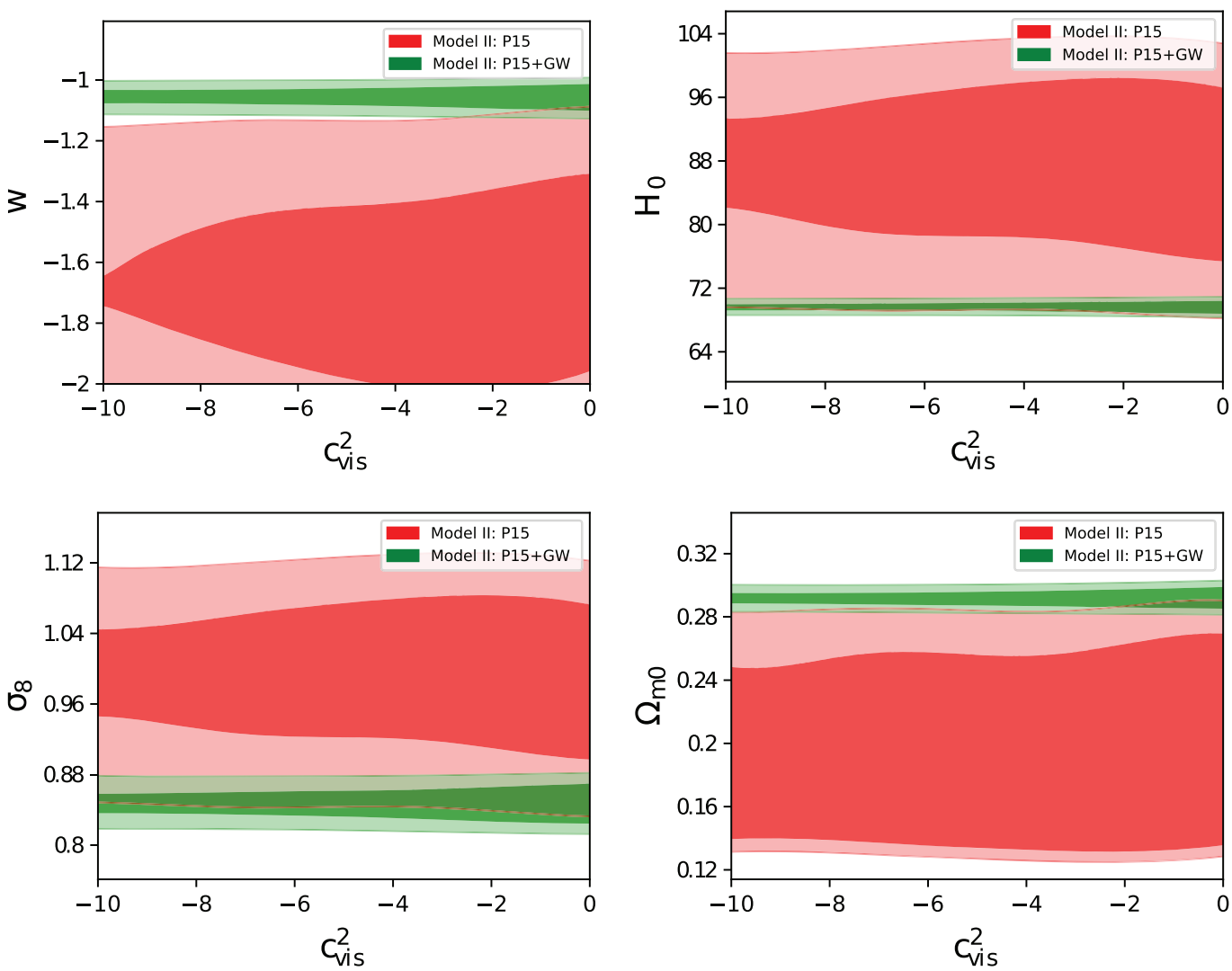

Figure 9. 2D contour plots for Model II showing the dependence of $c_{\mathrm{vis}}^{2}$ on other cosmological parameters for the P15 (red contours) and P15 + GW (green contours) data sets.
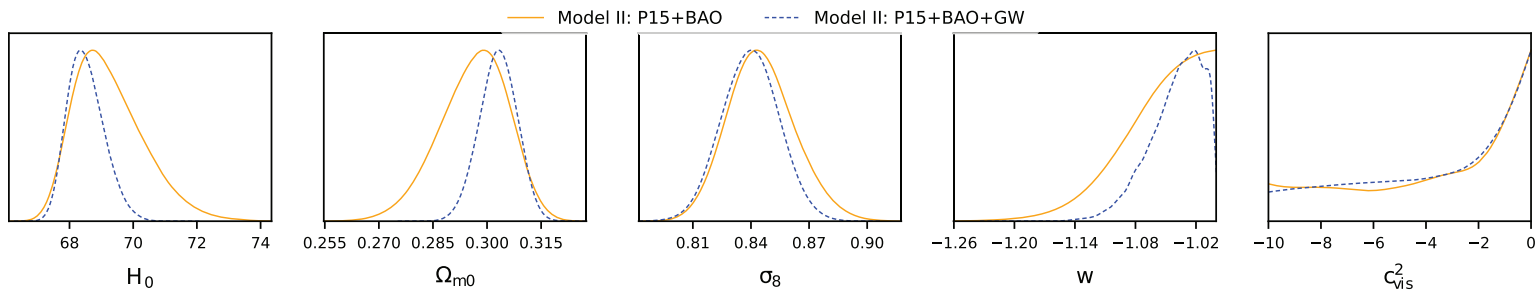

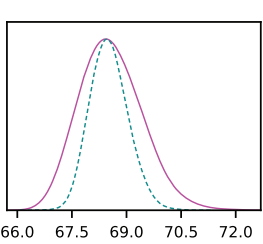

$\mathrm{H}_{0}$

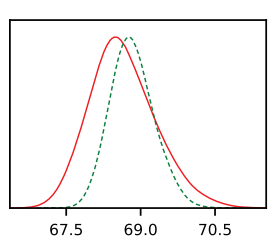

$\mathrm{H}_{0}$

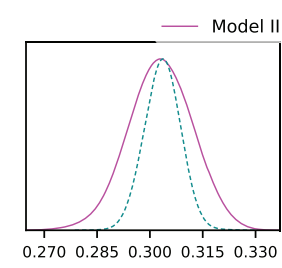

$\Omega_{\mathrm{m}}$

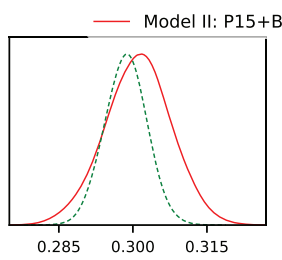

$\Omega_{\mathrm{m} 0}$

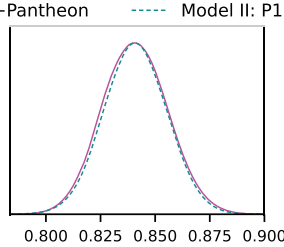

$\sigma_{8}$

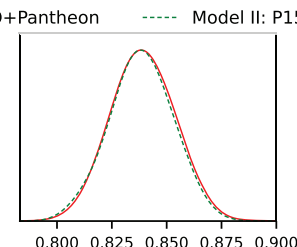

$\sigma_{8}$

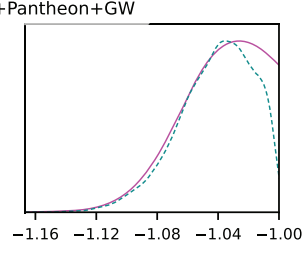

W

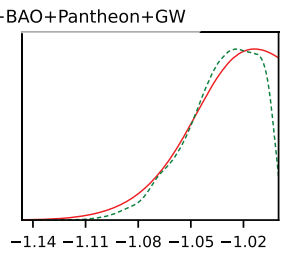

W
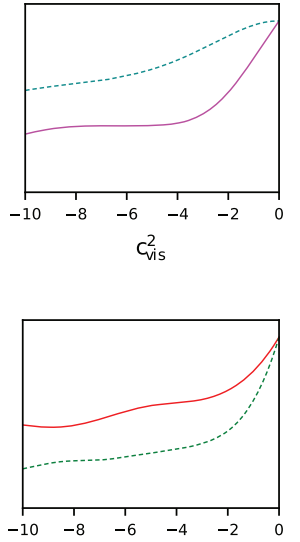

$\mathrm{c}_{\mathrm{vis}}^{2}$

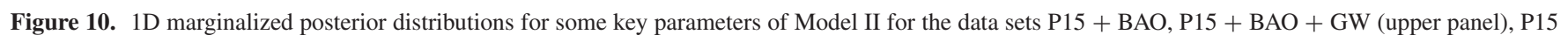
+ Pantheon, P15 + Pantheon + GW (middle panel), and P15 + BAO + Pantheon, P15 + BAO + Pantheon + GW (lower panel). 

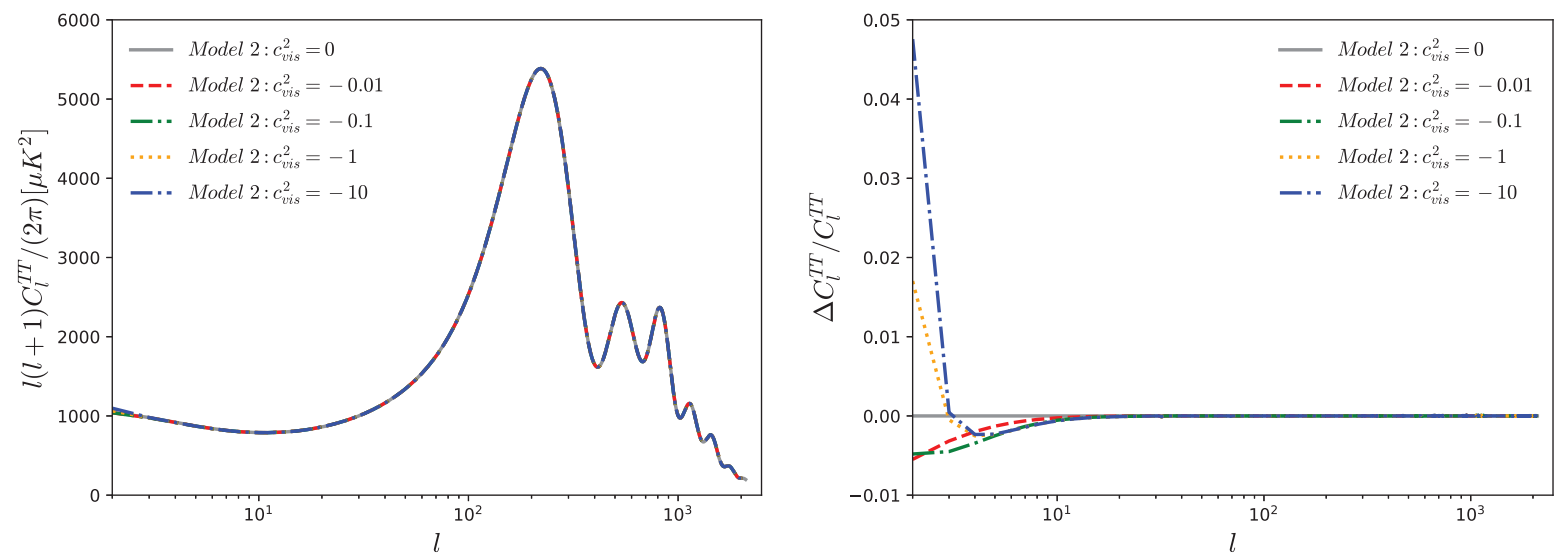

Figure 11. The plots reflect the effects on the CMB TT spectra for Model II taking various values of the viscous sound speed, $c_{\mathrm{vis}}^{2}$. As Model II restricts the DE equation of state, $w$ in the phantom region, so we fix $w=-1.1$ and the remaining parameters have been taken from the combined analysis P15+BAO + Pantheon (see the last column of Table 4). Note that the qualitative behaviour of the plots do not change if one varies the DE equation of state. From the right-hand panel, one can see that as long as $c_{\mathrm{vis}}^{2}$ increases, a very mild deviation of the curves with $c_{\mathrm{vis}}^{2} \neq 0$ from the curve representing $c_{\mathrm{vis}}^{2}=0$ (no anisotropic stress) appears.
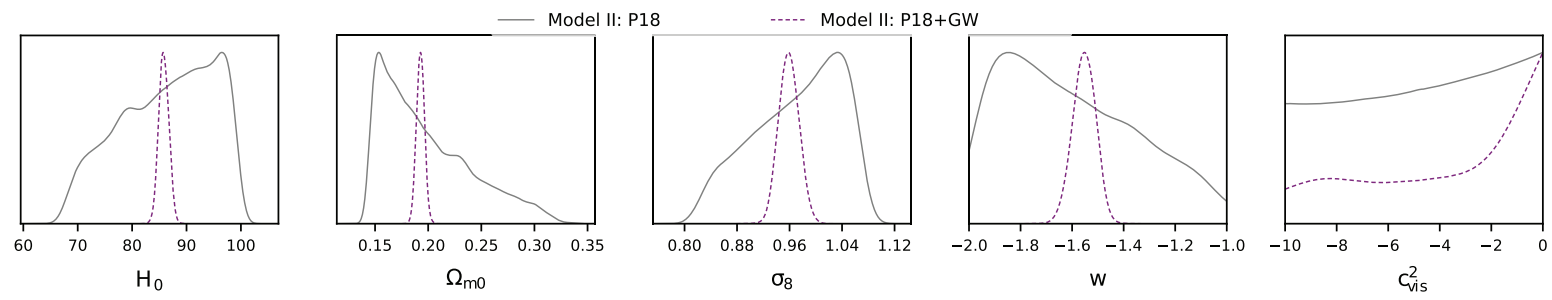

Figure 12. 1D marginalized posterior distributions for some parameters of Model II for the data sets P18 and P18 + GW.

Finally, similar to Model I, here also we consider the analyses with P18 and P18 + GW. The results are shown in Fig. 12. One can clearly see that P18 data are unable to constrain the parameter $c_{\mathrm{vis}}^{2}$, which is also unconstrained by the P15 data.

\section{CONCLUSION AND THE FINAL REMARKS}

In this paper, we have considered a very general cosmological framework in which the DE component has an anisotropic stress. Our aim is to constrain the anisotropic stress of DE in terms of the viscous sound speed $c_{\text {vis }}^{2}$ using the usual cosmological probes, namely CMB from Planck 2015 (P15), CMB from Planck 2018 (P18), BAO, and Pantheon, and then measure the constraining power of GW data. We have considered two different scenarios as follows: (i) Model I, where $c_{\mathrm{vis}}^{2}>0$ and $w>-1$, and (ii) Model II in which $c_{\mathrm{vis}}^{2}<0$ and $w<-1$.

We started our analyses with a number of cosmological probes, such as P15, P15 + BAO, P15 + Pantheon, and P15 + BAO + Pantheon and, afterwards, we measured the effects of the GW data from Einstein Telescope for the combinations P15 + GW, P15 + BAO + GW, P15 + Pantheon + GW, and P15 + BAO + Pantheon + GW. For Model I, the results of our analyses have been shown in Tables 2 (without GWSS) and 3 (with GWSS). In order to understand the effects of GWSS on the underlying scenario, we have shown the 1D posterior distributions of some key parameters in Figs 3 (only for P15 and P15 + GW) and 5 (for the remaining data sets). For Model II, Tables 4 (without GWSS) and 5 (with GWSS) summarize the main observational constraints on the parameters. In a similar fashion, for this model scenario, we have shown the 1D posterior distributions of some key parameters in Fig. 8 (only for the data sets P15 and P15 + GW) aiming to exhibit the constraining power of GW in Fig. 10. From the analyses, we find that the simulated GWSS data are able to provide stringent constraints on the cosmological parameters; specifically, we find GWSS data very powerful to constrain some of the key cosmological parameters of the scenarios, such as the Hubble constant, $H_{0}$, density parameter for the matter sector, $\Omega_{\mathrm{m} 0}$, and the DE equation of state, $w$. However, the parameter $c_{\mathrm{vis}}^{2}$ remains degenerate with every parameter of the model and this does not alter even after the inclusion of the GWSS data to the standard cosmological probes. This is pretty clear from all the plots (see e.g. Figs 3, 5, 8, and 10) shown in this paper. This is a striking and surprising fact of this work where, on one hand, we see that GW data are extremely effective to reduce the parameter space by reducing their error bars, while, on the other hand, this is not true for the viscous sound speed. We then employed the latest and final CMB data from Planck (labelled as P18) to analyse the models in order to see whether the new CMB data, i.e. P18, and also its combination with GW data, namely P18 + GW, could effectively constrain the parameter $c_{\text {vis }}^{2}$, which has been unconstrained for the previous data sets. We found that neither the final P18 data nor the combined data P18 $+\mathrm{GW}$ are able to constrain this parameter (see Fig. 7 for Model I and Fig. 12 for Model II). We already found that when P15 and P15 + GW data sets are unable to constrain $c_{\text {vis }}^{2}$, the inclusion of external data sets, such as, BAO, Pantheon, etc., does not add any extra constraining power beyond P15 and P15 + GW so that this parameter is constrained. Thus, it is clear that the inclusion of those 
external data sets to P18 will not be helpful in this case as well. Hence, novel and complementary astrophysical probes need to be found to probe the value of $c_{\text {vis }}^{2}$.

Interestingly, some complimentary probes have shown considerable progress in this direction. It Sapone et al. (2013) and Majerotto et al. (2016), the authors took an attempt to forecast the constraints on the viscous sound speed, $c_{\mathrm{vis}}^{2}$, using the Euclid and Planck surveys and found that the parameter $c_{\mathrm{vis}}^{2}$ can be well constrained using those future surveys. This is certainly a fascinating report since the usual cosmological probes are unable to constrain this viscous sound speed. On the other hand, recently using the model-independent approaches, the anistropic stress parameter has been confronted in presence of the observational data (Pinho et al. 2018; Arjona \& Nesseris 2020). The results in Pinho et al. (2018) and Arjona \& Nesseris 2020) strongly suggesting that a non-zero value of the anisotropic stress parameter is supported by recent observations and this consequently implies either the modification of underlying gravitational theory or the existence of an imperfect DE fluid clustering at subhorizon scales. Additionally, one can also model the anisotropic stress linked either with the DE density or DM density as in Cardona et al. (2014), and within such scenarios, the underlying parameters quantifying the anisotropic stress in DE can be well constrained as least using the CMB data Planck as well as other cosmological probes; see Cardona et al. (2014) for details.

However, the investigations are not over and the cosmic picture is still not perfectly clear. Although the future cosmological survey Euclid (Laureijs et al. 2011; Scaramella et al. 2014) has played a crucial role (Sapone et al. 2013; Majerotto et al. 2016), other upcoming surveys are equally important for a better picture on the DE anisotropic stress. Thus, we believe that CMB Stage-4 (Abitbol et al. 2017), Dark Energy Spectroscopic Instrument (DESI; DESI Collaboration et al. 2016), Large Synoptic Survey Telescope (LSST; Hložek et al. 2019; Mandelbaum et al. 2019; Newman et al. 2019), and Simons Observatory (Ade et al. 2019) should be employed in this framework for a clear visualization on this scenario. Such an analysis is left for a future work.

\section{ACKNOWLEDGEMENTS}

The authors thank the referee for useful comments that helped us to improve the quality of discussion of this paper. WY was supported by the National Natural Science Foundation of China under Grants No. 11705079 and No. 11647153 . SP has been supported by the Mathematical Research Impact-Centric Support Scheme (MATRICS), File No. MTR/2018/000940, given by the Science and Engineering Research Board (SERB), Govt. of India. DFM thanks the Research Council of Norway and the NOTUR computing facilities. MD acknowledges the support from the National Natural Science Foundation of China under Grant No. 11675032.

\section{DATA AVAilability}

In this work, we have used standard cosmological probes such as cosmic microwave background radiation from Planck 2015 and 2018 , Pantheon sample of the SNe Ia, baryon acoustic oscillations distance measurements from different astronomical missions, and the luminosity distance measurements from $\mathcal{O}\left(10^{3}\right)$ mock GW events using Einstein Telescope. The standard cosmological probes are publicly available and one can easily get them just by typing the name of the observational missions that are properly cited in the main text. Concerning the mock data, one can easily generate it assuming any fiducial cosmological model. However, if anyone faces any difficulties to generate this data set, please send us an e-mail.

\section{REFERENCES}

Abbott B. P. et al., 2016a, Phys. Rev. Lett., 116, 061102

Abbott B. P. et al., 2016b, Phys. Rev. Lett., 116, 241103

Abbott B. P. et al., 2017a, Phys. Rev. Lett., 118, 221101

Abbott B. P. et al., 2017b, Phys. Rev. Lett., 119, 141101

Abbott B. P. et al., 2017c, ApJ, 851, L35

Abitbol M. H. et al., 2017, preprint (arXiv:1706.02464)

Adam R. et al., 2016, A\&A, 594, A1

Addazi A., Cai Y.-F., Marciano A., 2018, Phys. Lett. B, 782, 732

Ade P. A. R. et al., 2016, A\&A, 594, A13

Ade P. et al., 2019, J. Cosmol. Astropart. Phys., 1902, 056

Amaro-Seoane P. et al., 2017, preprint (arXiv:1702.00786)

Amendola L., 2000, Phys. Rev. D, 62, 043511

Amendola L., Fogli S., Guarnizo A., Kunz M., Vollmer A., 2014, Phys. Rev. D, 89, 063538

Arjona R., Nesseris S., 2020, preprint (arXiv:2001.11420)

Arjona R., Cardona W., Nesseris S., 2019, Phys. Rev. D, 99, 043516

Armendariz-Picon C., 2004, J. Cosmol. Astropart. Phys., 0407, 007

Armendariz-Picon C., Mukhanov V. F., Steinhardt P. J., 2001, Phys. Rev. D, 63, 103510

Bachega R. R. A., Costa A. A., Abdalla E., Fornazier K. S. F., 2020, J. Cosmol. Astropart. Phys., 05, 021

Bagla J. S., Jassal H. K., Padmanabhan T., 2003, Phys. Rev. D, 67, 063504

Baker T., Bellini E., Ferreira P. G., Lagos M., Noller J., Sawicki I., 2017, Phys. Rev. Lett., 119, 251301

Bean R., Dore O., 2004, Phys. Rev. D, 69, 083503

Belgacem E. et al., 2019, J. Cosmol. Astropart. Phys., 1907, 024

Bettoni D., Ezquiaga J. M., Hinterbichler K., Zumalacárregui M., 2017, Phys. Rev. D, 95, 084029

Beutler F. et al., 2011, MNRAS, 416, 3017 
Bonilla A., D’Agostino R., Nunes R. C., de Araujo J. C. N., 2020, J. Cosmol. Astropart. Phys., 03, 015

Brevik I. H., Nojiri S., Odintsov S. D., Vanzo L., 2004, Phys. Rev. D, 70, 043520

Cai R.-G., Yang T., 2017, Phys. Rev. D, 95, 044024

Cai Y.-F., Zhang X., 2016, Sci. China Phys. Mech. Astron., 59, 670431

Cai Y.-F., Li C., Saridakis E. N., Xue L., 2018, Phys. Rev. D, 97, 103513

Capozziello S., De Laurentis M., 2011, Phys. Rep., 509, 167

Cardona W., Hollenstein L., Kunz M., 2014, J. Cosmol. Astropart. Phys., 07, 032

Casalino A., Rinaldi M., Sebastiani L., Vagnozzi S., 2018, Phys. Dark Universe, 22, 108

Casalino A., Rinaldi M., Sebastiani L., Vagnozzi S., 2019, Class. Quantum Gravity, 36, 017001

Chakraborty S., Chakravarti K., Bose S., SenGupta S., 2018, Phys. Rev. D, 97, 104053

Chang B., Xu L., 2014, Phys. Rev. D, 90, 027301

Chang B., Lu J., Xu L., 2014, Phys. Rev. D, 90, 103528

Chassande-Mottin E., Hendry M., Sutton P. J., Marka S., 2011, Gen. Relativ. Gravit., 43, 437

Copeland E. J., Sami M., Tsujikawa S., 2006, Int. J. Mod. Phys. D, 15, 1753

Copeland E. J., Kopp M., Padilla A., Saffin P. M., Skordis C., 2019, Phys. Rev. Lett., 122, 061301

Creminelli P., Vernizzi F., 2017, Phys. Rev. Lett., 119, 251302

D’Agostino R., Nunes R. C., 2019, Phys. Rev. D, 100, 044041

DESI Collaboration et al., 2016, preprint (arXiv:1611.00036)

Di Valentino E., Melchiorri A., 2018, Phys. Rev. D, 97, 041301

Di Valentino E., Holz D. E., Melchiorri A., Renzi F., 2018, Phys. Rev. D, 98, 083523

Du M., Yang W., Xu L., Pan S., Mota D. F., 2019, Phys. Rev. D, 100, 043535

Ezquiaga J. M., Zumalacárregui M., 2017, Phys. Rev. Lett., 119, 251304

Flauger R., Weinberg S., 2018, Phys. Rev. D, 97, 123506

Gair J. R., Mandel I., Miller M. C., Volonteri M., 2011, Gen. Relativ. Gravit., 43, 485

Gelman A., Rubin D., 1992, Stat. Sci., 7, 457

Gibbons G. W., 2002, Phys. Lett., B537, 1

Gil-Marín H. et al., 2016, MNRAS, 460, 4210

Hannam M., Hawke I., 2011, Gen. Relativ. Gravit., 43, 465

Hložek R. A. et al., 2019, Bull. Am. Astron. Soc., 51, 369

Hu W., 1998, ApJ, 506, 485

Huerta E. A., Gair J. R., 2011a, Phys. Rev. D, 83, 044020

Huerta E. A., Gair J. R., 2011b, Phys. Rev. D, 83, 044021

Huey G., 2004, preprint (astro-ph/0411102)

Kase R., Tsujikawa S., 2019, Int. J. Mod. Phys. D, 28, 1942005

Kawamura S. et al., 2011, Class. Quantum Gravity, 28, 094011

Kiselev V. V., 2004, Class. Quantum Gravity, 21, 3323

Koivisto T., Mota D. F., 2006, Phys. Rev. D, 73, 083502

Koivisto T., Mota D. F., 2007a, Phys. Rev. D, 75, 023518

Koivisto T., Mota D. F., 2007b, Phys. Lett. B, 644, 104

Laureijs R. et al., 2011, preprint (arXiv:1110.3193)

Lewis A., 2013, Phys. Rev. D, 87, 103529

Lewis A., Bridle S., 2002, Phys. Rev. D, 66, 103511

Lin K. et al., 2019, Phys. Rev. D, 99, 023010

Liu T. et al., 2018, Phys. Rev. D, 98, 083023

Luo J. et al., 2016, Class. Quantum Gravity, 33, 035010

Majerotto E., Sapone D., Schäfer B. M., 2016, MNRAS, 456, 109

Ma C.-P., Bertschinger E., 1995, ApJ, 455, 7

Mandelbaum R. et al., 2019, Bull. Am. Astron. Soc., 51, 363

Maselli A., Marassi S., Ferrari V., Kokkotas K., Schneider R., 2016, Phys. Rev. Lett., 117, 091102

Mishra C. K., Arun K. G., Iyer B. R., Sathyaprakash B. S., 2010, Phys. Rev. D, 82, 064010

Mota D. F., Shaw D. J., 2006, Phys. Rev. Lett., 97, 151102

Mota D. F., Shaw D. J., 2007, Phys. Rev. D, 75, 063501

Mota D. F., van de Bruck C., 2004, A\&A, 421, 71

Mota D. F., Kristiansen J. R., Koivisto T., Groeneboom N. E., 2007, MNRAS, 382, 793

Newman J. A. et al., 2019, preprint (arXiv:1903.09325)

Nojiri S., Odintsov S. D., 2011, Phys. Rep., 505, 59

Nojiri S., Odintsov S. D., Oikonomou V. K., 2017, Phys. Rep., 692, 1

Nunes R. C., Pan S., Saridakis E. N., 2018, Phys. Rev., 98, 104055

Nunes R. C., Alves M. E. S., de Araujo J. C. N., 2019, Phys. Rev. D, 100, 064012

Oost J., Mukohyama S., Wang A., 2018, Phys. Rev. D, 97, 124023

Pinho A. M., Casas S., Amendola L., 2018, J. Cosmol. Astropart. Phys., 11, 027

Planck Collaboration et al., 2016, A\&A, 594, A11

Planck Collaboration et al., 2018, A\&A, preprint (arXiv:1807.06210)

Planck Collaboration et al., 2019, preprint (arXiv:1907.12875)

Punturo M. et al., 2010, Class. Quantum Gravity, 27, 194002

Qiao J., Zhu T., Zhao W., Wang A., 2019, Phys. Rev. D, 100, 124058

Regimbau T. et al., 2012, Phys. Rev. D, 86, 122001

Ross A. J., Samushia L., Howlett C., Percival W. J., Burden A., Manera M., 2015, MNRAS, 449, 835 
Saltas I. D., Kunz M., 2011, Phys. Rev. D, 83, 064042

Sapone D., Majerotto E., Kunz M., Garilli B., 2013, Phys. Rev. D, 88, 043503

Sathyaprakash B. et al., 2012, Class. Quantum Gravity, 29, 124013

Sathyaprakash B. S., Schutz B. F., Van Den Broeck C., 2010, Class. Quantum Gravity, 27, 215006

Scaramella R. et al., 2014, in Heavens A. F., Starck J.-L., eds, Proc. IAU Symp. 306, Statistical Challenges in 21st Century Cosmology. Kluwer, Dordrecht, p. 375

Schimd C. et al., 2007, A\&A, 463, 405

Scolnic D. M. et al., 2018, ApJ, 859, 101

Sen A., 2002a, J. High Energy Phys., 04, 048

Sen A., 2002b, J. High Energy Phys., 07, 065

Sen A., 2002c, Mod. Phys. Lett. A, 17, 1797

Sen A., 2003, Int. J. Mod. Phys. A, 18, 4869

Sen A., 2005, Phys. Scr., T117, 70

Shafieloo A., Keeley R. E., Linder E. V., 2020, J. Cosmol. Astropart. Phys., 03, 019

Taylor S. R., Gair J. R., 2012, Phys. Rev. D, 86, 023502

Visinelli L., Bolis N., Vagnozzi S., 2018, Phys. Rev. D, 97, 064039

Wei J.-J., 2018, ApJ, 868, 29

Wetterich C., 1988, Nucl. Phys. B, 302, 668

Xia J.-Q., Cai Y.-F., Qiu T.-T., Zhao G.-B., Zhang X., 2008, Int. J. Mod. Phys. D, 17, 1229

Yang W., Pan S., Xu L., Mota D. F., 2019a, MNRAS, 482, 1858

Yang W., Vagnozzi S., Di Valentino E., Nunes R. C., Pan S., Mota D. F., 2019b, J. Cosmol. Astropart. Phys., 1907, 037

Yang W., Pan S., Di Valentino E., Wang B., Wang A., 2020, J. Cosmol. Astropart. Phys., 05, 050

Zhang X. et al., 2018, preprint (arXiv:1806.02581)

Zhang X., Yu J., Liu T., Zhao W., Wang A., 2017, Phys. Rev. D, 95, 124008

Zhang J.-F., Dong H.-Y., Qi J.-Z., Zhang X., 2020, Eur. Phys. J. C., , 80, 217

Zhang J.-F., Zhang M., Jin S.-J., Qi J.-Z., Zhang X., 2019, J. Cosmol. Astropart. Phys., 1909, 068

Zhao W., Van Den Broeck C., Baskaran D., Li T. G. F., 2011, Phys. Rev. D, 83, 023005

Zhao W., Wright B. S., Li B., 2018, J. Cosmol. Astrophys. Phys., 1810, 052

Zhao W., Liu T., Wen L., Zhu T., Wang A., Hu Q., Zhou C., 2019a, Eur. Phys. J. C, preprint (arXiv:1909.13007)

Zhao X. et al., 2019b, Phys. Rev. D, 100, 083012

Zhao W., Zhu T., Qiao J., Wang A., 2020, Phys. Rev. D, 101, 024002

This paper has been typeset from a $\mathrm{T}_{\mathrm{E}} \mathrm{X} / \mathrm{L} \mathrm{A} \mathrm{E} \mathrm{X}$ file prepared by the author. 